\title{
Transversity generalized parton distributions for the deuteron
}

\author{
W. Cosyn ${ }^{1, *}$ and B. Pire ${ }^{2}$ \\ ${ }^{1}$ Department of Physics and Astronomy, Ghent University, Proeftuinstraat 86, 89000 Ghent, Belgium \\ ${ }^{2}$ Centre de Physique Théorique, École Polytechnique, CNRS, 91128 Palaiseau, France
}

(Received 13 June 2018; published 22 October 2018)

\begin{abstract}
Transversity generalized parton distributions (GPDs) appear as scalar functions in the decomposition of off-forward quark-quark and gluon-gluon correlators with a parton helicity flip. For a spin 1 hadron, we find nine transversity GPDs for both quarks and gluons at leading twist 2 . We study these twist-2 chiral odd quark transversity GPDs for the deuteron in a light cone convolution model, based on the impulse approximation, and using the lowest Fock-space state for the deuteron.
\end{abstract}

DOI: $10.1103 /$ PhysRevD.98.074020

\section{INTRODUCTION}

The factorization of hard exclusive amplitudes in the generalized Bjorken regime [1,2] as the convolution of generalized parton distributions (GPDs) with perturbatively calculable coefficient functions allows us to get access to the three-dimensional structure of nucleons or nuclei through the extraction of the various quark and gluon GPDs. The connection between GPDs and parton-hadron helicity amplitudes allows an easy counting of twist-2 GPDs: there are $2(2 J+1)^{2}$ GPDs for each quark flavor (or for the gluon) in a nucleus of spin $J$. Half of these GPDs correspond to parton helicity nonflip, the other half-which are dubbed transversity GPDs-correspond to parton helicity flip. In the quark case, the helicity nonflip GPDs are chiral even, while the helicity flip GPDs are chiral odd. The helicity flip and nonflip sectors evolve independently in the renormalization scale. Moreover, the quark and gluon sectors do not mix in the evolution of transversity GPDs.

Nuclear GPDs [3-12] obey the same rules as nucleon GPDs and are accessible through coherent exclusive processes which may be isolated from incoherent processes where the target nucleus breaks during the hard interaction. As the simplest composite nucleus, the deuteron is a fascinating object to scrutinize in order to understand the QCD confinement mechanism [13]. The study of hard reactions which allow us to access its quark and gluon structure is at the heart of the on-going physics program at Jefferson Lab (JLab) as well as the future electron-ion collider (EIC) program. The study of the deuteron GPDs

\footnotetext{
*wim.cosyn@ugent.be
}

Published by the American Physical Society under the terms of the Creative Commons Attribution 4.0 International license. Further distribution of this work must maintain attribution to the author(s) and the published article's title, journal citation, and DOI. Funded by SCOAP. should allow to understand more deeply the relation between the deuteron and nucleon structures. The spin 1 nature of the deuteron makes it a particularly rich object from the point of view of building the spin from the constituent spins and orbital angular momenta.

Contrarily to the nucleon GPDs which have been the subject of many works - both theoretically and experimentallythe study of deuteron GPDs is still in its infancy; its founding blocks are the definition of helicity nonflip quark and gluon GPDs [14] and the calculation of deeply virtual Compton scattering (DVCS) and deep exclusive meson production (DEMP) amplitudes [15,16] in the coherent reactions on a deuteron. First results on coherent hard exclusive reactions have been obtained at JLab [17]. In the present paper, we study the transversity sector of deuteron twist-2 GPDs which was left aside up to now.

The paper is organized as follows. The transversity GPDs of spin 1 hadrons are the objects of study in Sec. II: we start with introducing the necessary kinematic variables in Sec. II A, list the general correlators and their symmetry properties in Sec. II B, and subsequently introduce the transversity GPDs for spin 1 and comment on their properties for quarks (Sec. II C) and gluons (Sec. II D). In the following Sec. III, we outline the convolution formalism for the deuteron, with kinematic variables defined in Sec. III A, the deuteron light-front wave function and chiral odd nucleon GPDs discussed in Secs. III B and III C, and finally the convolution model is presented in Sec. III D. Results obtained in the convolution formalism for transversity helicity amplitudes and GPDs in the quark sector are discussed in Sec. IV, and sum rules from the first moments of the quark transversity GPDs are covered in Sec. V. Conclusions are stated in Sec. VI. The notation, sign and normalization conventions used throughout this article are summarized in Appendix A, while Appendix B contains a summary of the properties of parity and time reversal symmetries on the light front. The relations 
between transversity helicity amplitudes and GPDs for spin 1 hadrons are listed in Appendix C, and a minimal convolution model used to obtain some analytical results is outlined in Appendix D.

We shall not deal with the phenomenology of these GPDs in this article and leave this topic for further work. Similarly, the polynomiality properties of spin 1 GPDs and the connection between general moments and the generalized form factors will be discussed elsewhere. At present, no parametrization for the nucleon gluon transversity GPDs is available [18]. Consequently, in this article we do not consider calculations in the convolution model for the deuteron gluon transversity GPDs.

\section{TRANSVERSITY GPDS FOR SPIN 1 HADRONS}

The central objects that define GPDs are Fourier transforms of gauge-invariant off-forward parton correlators, where the initial (final) hadron in the correlator matrix element has four-momentum $p\left(p^{\prime}\right)$, light-front helicity $\lambda\left(\lambda^{\prime}\right)$ and mass $M$. For quarks these correlators take the form

$$
\left\langle p^{\prime} \lambda^{\prime}|\bar{\psi}(-\kappa n) \Gamma \psi(\kappa n)| p \lambda\right\rangle,
$$

with $\Gamma$ a general Dirac structure, and the two quark fields are separated along a lightlike four-vector $n^{\mu}\left(n^{2}=0\right)$. In this work, we use the light cone gauge $(n A)=0$, so no explicit Wilson lines appear in the correlators. Similar correlators can also be introduced for gluons (see Sec. II B). These objects encode long distance, strongly coupled QCD dynamics and can be diagramatically represented by the blob in Fig. 1.

\section{A. Kinematical variables}

We introduce the standard kinematic variables for these matrix elements, being the average hadron momentum $P$, momentum transfer $\Delta$, skewness $\xi$ (which determines the longitudinal momentum transfer) and $t$,

$$
\begin{gathered}
P=\frac{p+p^{\prime}}{2}, \quad \Delta=p^{\prime}-p, \\
t=\Delta^{2}, \quad \xi=-\frac{(\Delta n)}{2(P n)} .
\end{gathered}
$$

Depending on the skewness $\xi$, the momentum transfer squared $t$ (which is negative) has a maximum value

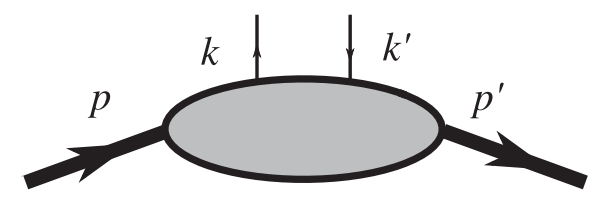

FIG. 1. Diagrammatic representation of an off-forward parton correlator.

$$
t_{0}=-\frac{4 M^{2} \xi^{2}}{1-\xi^{2}},
$$

and we can write

$$
t_{0}-t=-2 M^{2} \frac{1+\xi^{2}}{1-\xi^{2}}+2\left(p p^{\prime}\right)
$$

The four-vector $2 \xi P+\Delta$ is orthogonal to $n[((2 \xi P+\Delta) n)=$ $0]$ and has the norm

$$
(2 \xi P+\Delta)^{2}=-\left(1-\xi^{2}\right)\left(t_{0}-t\right) .
$$

The following combination of kinematic variables occurs a lot in formulas in this work, so an extra dimensionless variable is defined:

$$
D \equiv \frac{\sqrt{\left(t_{0}-t\right)\left(1-\xi^{2}\right)}}{2 M}
$$

As we study parton correlators for spin 1 particles, we consider a basis of three polarization four-vectors, both for the initial (unprimed four-vectors) and final (primed four-vectors) spin 1 hadron state [14], normalized to $\left(\epsilon^{*(i)} \epsilon^{(i)}\right)=-1$ and orthogonal to the particle fourmomentum $\left[(\epsilon(i) p)=\left(\epsilon^{\prime}(i) p^{\prime}\right)=0\right]{ }^{1}$

$$
\begin{aligned}
\epsilon^{(0) \mu}= & \frac{1}{M}\left(p^{\mu}-\frac{M^{2}}{1+\xi} \frac{n^{\mu}}{(P n)}\right), \\
\epsilon^{\prime(0) \mu}= & \frac{1}{M}\left(p^{\prime \mu}-\frac{M^{2}}{1-\xi} \frac{n^{\mu}}{(P n)}\right), \\
\epsilon^{(1) \mu}= & -\frac{1}{\sqrt{\left(1-\xi^{2}\right)\left(t_{0}-t\right)}}\left((1+\xi) p^{\prime \mu}-(1-\xi) p^{\mu}\right. \\
& \left.-\frac{\xi\left(t_{0}-t\right)-t_{0}}{2 \xi} \frac{n^{\mu}}{(P n)}\right), \\
\epsilon^{\prime(1) \mu}= & -\frac{1}{\sqrt{\left(1-\xi^{2}\right)\left(t_{0}-t\right)}}\left((1+\xi) p^{\prime \mu}-(1-\xi) p^{\mu}\right. \\
& \left.+\frac{\xi\left(t_{0}-t\right)+t_{0}}{2 \xi} \frac{n^{\mu}}{(P n)}\right), \\
\epsilon^{(2) \mu}= & \epsilon^{\prime(2) \mu}=\frac{1}{\sqrt{\left(1-\xi^{2}\right)\left(t_{0}-t\right)}} \frac{\varepsilon^{\mu \nu \alpha \beta} p_{\nu}^{\prime} p_{\alpha} n_{\beta}}{(P n)} .
\end{aligned}
$$

We use

$$
\begin{aligned}
\epsilon(0) & =\epsilon^{(0)}, \\
\epsilon( \pm) & =\mp e^{ \pm i \phi}\left(\epsilon^{(1)} \pm i \epsilon^{(2)}\right) / \sqrt{2},
\end{aligned}
$$

\footnotetext{
${ }^{1}$ Our sign convention for the Levi-Civita tensor and other quantities is summarized in Appendix A.
} 
as definite light cone helicity polarization four-vectors for the initial hadron, and similar expressions for the primed polarization four-vectors and the final hadron. In Eq. (8), $\phi$ is the azimuthal angle of the four-vector $\Delta+2 \xi P$.

\section{B. Correlators and symmetry properties}

The following quark-quark correlators determine the leading twist-2 quark GPDs $[1,2,19]$ :

$$
\begin{aligned}
V_{\lambda^{\prime} \lambda}^{q} & =\int \frac{d \kappa}{2 \pi} e^{2 i x \kappa(P n)}\left\langle p^{\prime} \lambda^{\prime}|\bar{\psi}(-\kappa n)(\gamma n) \psi(\kappa n)| p \lambda\right\rangle, \\
A_{\lambda^{\prime} \lambda}^{q} & =\int \frac{d \kappa}{2 \pi} e^{2 i x \kappa(P n)}\left\langle p^{\prime} \lambda^{\prime}\left|\bar{\psi}(-\kappa n) \gamma_{5}(\gamma n) \psi(\kappa n)\right| p \lambda\right\rangle, \\
T_{\lambda^{\prime} \lambda}^{q i} & =\int \frac{d \kappa}{2 \pi} e^{2 i x \kappa(P n)}\left\langle p^{\prime} \lambda^{\prime}\left|\bar{\psi}(-\kappa n)\left(i n_{\mu} \sigma^{\mu i}\right) \psi(\kappa n)\right| p \lambda\right\rangle,
\end{aligned}
$$

where $i$ is a transverse index and transverse is relative to the lightlike four-vectors $n$ and $\bar{n}\left(n^{2}=\bar{n}^{2}=0, n \bar{n}=1\right)$. The decomposition for the first two (vector $V_{\lambda^{\prime} \lambda}^{q}$, axial vector $\left.A_{\lambda^{\prime} \lambda}^{q}\right)$ was considered for spin 1 hadrons in Ref. [14] and determines the nine chiral even quark GPDs for spin 1 (five for $V_{\lambda^{\prime} \lambda}^{q}$, four for $A_{\lambda^{\prime} \lambda}^{q}$ ). The decomposition of the tensor correlator $T_{\lambda^{\prime} \lambda}^{q i}$ is given below (Sec. II C) and determines nine spin 1 chiral odd quark GPDs.

Similarly, the following gluon-gluon correlators determine the leading twist-2 gluon GPDs $[1,2,19]$ :

$$
\begin{aligned}
V_{\lambda^{\prime} \lambda}^{g}= & \frac{2}{(P n)} \int \frac{d \kappa}{2 \pi} e^{2 i x \kappa(P n)} \\
& \times\left\langle p^{\prime} \lambda^{\prime}\left|\operatorname{Tr}\left[n_{\alpha} G^{\alpha \mu}(-\kappa n) G_{\beta \mu}(\kappa n) n^{\beta}\right]\right| p \lambda\right\rangle \\
= & \frac{1}{(P n)} \int \frac{d \kappa}{2 \pi} e^{2 i x \kappa(P n)}\left\langle p^{\prime} \lambda^{\prime}\right| \operatorname{Tr}\left[n _ { \alpha } \left(G^{\alpha R}(-\kappa n) G^{\beta L}(\kappa n)\right.\right. \\
& \left.\left.+G^{\alpha L}(-\kappa n) G^{\beta R}(\kappa n)\right) n_{\beta}\right]|p \lambda\rangle, \\
A_{\lambda^{\prime} \lambda}^{g}= & -\frac{2 i}{(P n)} \int \frac{d \kappa}{2 \pi} e^{2 i x \kappa(P n)} \\
& \times\left\langle p^{\prime} \lambda^{\prime}\left|\operatorname{Tr}\left[n_{\alpha} G^{\alpha \mu}(-\kappa n) G_{\beta \mu}(\kappa n) n^{\beta}\right]\right| p \lambda\right\rangle \\
= & \frac{1}{(P n)} \int \frac{d \kappa}{2 \pi} e^{2 i x \kappa(P n)}\left\langle p^{\prime} \lambda^{\prime}\right| \operatorname{Tr}\left[n _ { \alpha } \left(G^{\alpha R}(-\kappa n) G^{\beta L}(\kappa n)\right.\right. \\
& \left.\left.-G^{\alpha L}(-\kappa n) G^{\beta R}(\kappa n)\right) n_{\beta}\right]|p \lambda\rangle, \\
T_{\lambda^{\prime} \lambda}^{g i j}= & -\frac{2}{(P n)} \int \frac{d \kappa}{2 \pi} e^{2 i x \kappa(P n)} \\
& \times\left\langle p^{\prime} \lambda^{\prime}\left|\operatorname{Tr} \hat{S}\left[n_{\alpha} G^{\alpha i}(-\kappa n) n_{\beta} G^{\beta j}(\kappa n)\right]\right| p \lambda\right\rangle,
\end{aligned}
$$

where $i, j$ are transverse indices, the operator $\hat{S}$ implies symmetrization and removal of trace, and transverse fourvector components $a^{R / L}$ are defined as

$$
\begin{aligned}
& a^{R}=a^{x}+i a^{y}, \\
& a^{L}=a^{x}-i a^{y} .
\end{aligned}
$$

Again, the decomposition of $V_{\lambda^{\prime} \lambda}^{q}, A_{\lambda^{\prime} \lambda}^{q}$ for spin 1 hadrons has been discussed earlier [14], and the composition of the tensor correlator $T_{\lambda^{\prime} \lambda}^{g i j}$ is given below in Sec. II D.

As $T_{\lambda^{\prime} \lambda}^{g R L}=T_{\lambda^{\prime} \lambda}^{g L R}=0$, there remain two independent matrix elements for the tensor gluon-gluon correlator,

$$
\begin{aligned}
T_{\lambda^{\prime} \lambda}^{g R R}= & -\frac{2}{(P n)} \int \frac{d \kappa}{2 \pi} e^{2 i x \kappa(P n)} \\
& \times\left\langle p^{\prime} \lambda^{\prime}\left|\operatorname{Tr}\left[n_{\alpha} G^{\alpha R}(-\kappa n) n_{\beta} G^{\beta R}(\kappa n)\right]\right| p \lambda\right\rangle, \\
T_{\lambda^{\prime} \lambda}^{g L L}= & -\frac{2}{(P n)} \int \frac{d \kappa}{2 \pi} e^{2 i x \kappa(P n)} \\
& \times\left\langle p^{\prime} \lambda^{\prime}\left|\operatorname{Tr}\left[n_{\alpha} G^{\alpha L}(-\kappa n) n_{\beta} G^{\beta L}(\kappa n)\right]\right| p \lambda\right\rangle .
\end{aligned}
$$

Hermiticity and discrete light-front symmetries ${ }^{2}$ impose the following constraints on the correlators ${ }^{3}$ :

(i) Hermiticity

$$
\begin{aligned}
V_{\lambda^{\prime} \lambda}^{*}(\Delta, P, n) & =V_{\lambda \lambda^{\prime}}(-\Delta, P, n), \\
A_{\lambda^{\prime} \lambda}^{*}(\Delta, P, n) & =A_{\lambda \lambda^{\prime}}(-\Delta, P, n), \\
T_{\lambda^{\prime} \lambda}^{q R / L *}(\Delta, P, n) & =-T_{\lambda \lambda^{\prime}}^{q L / R}(-\Delta, P, n), \\
T_{\lambda^{\prime} \lambda}^{g R R *}(\Delta, P, n) & =T_{\lambda \lambda^{\prime}}^{g L L}(-\Delta, P, n) .
\end{aligned}
$$

(ii) Light-front parity $\mathcal{P}_{\perp}$

$$
\begin{aligned}
V_{\lambda^{\prime} \lambda}(\Delta, P, n) & =V_{-\lambda^{\prime}-\lambda}(\tilde{\Delta}, \tilde{P}, \tilde{n}), \\
A_{\lambda^{\prime} \lambda}(\Delta, P, n) & =-A_{-\lambda^{\prime}-\lambda}(\tilde{\Delta}, \tilde{P}, \tilde{n}), \\
T_{\lambda^{\prime} \lambda}^{q R / L}(\Delta, P, n) & =-T_{-\lambda^{\prime}-\lambda}^{q L / R}(\tilde{\Delta}, \tilde{P}, \tilde{n}), \\
T_{\lambda^{\prime} \lambda}^{g R R}(\Delta, P, n) & =T_{-\lambda^{\prime}-\lambda}^{g L L}(\tilde{\Delta}, \tilde{P}, \tilde{n}) .
\end{aligned}
$$

(iii) Light-front time reversal $\mathcal{T}_{\perp}$

$$
\begin{aligned}
V_{\lambda^{\prime} \lambda}(\Delta, P, n) & =(-1)^{\lambda^{\prime}-\lambda} V_{\lambda \lambda^{\prime}}(-\tilde{\Delta}, \tilde{P}, \tilde{n}), \\
A_{\lambda^{\prime} \lambda}(\Delta, P, n) & =(-1)^{\lambda^{\prime}-\lambda} A_{\lambda \lambda^{\prime}}(-\tilde{\Delta}, \tilde{P}, \tilde{n}), \\
T_{\lambda^{\prime} \lambda}^{q R / L}(\Delta, P, n) & =(-1)^{\lambda^{\prime}-\lambda} T_{\lambda \lambda^{\prime}}^{q L / R}(-\tilde{\Delta}, \tilde{P}, \tilde{n}), \\
T_{\lambda^{\prime} \lambda}^{g R R}(\Delta, P, n) & =(-1)^{\lambda^{\prime}-\lambda} T_{\lambda \lambda^{\prime}}^{g L L}(-\tilde{\Delta}, \tilde{P}, \tilde{n}) .
\end{aligned}
$$

\footnotetext{
${ }^{2}$ The properties of light-front parity and time reversal are summarized in Appendix B.

${ }^{3}$ If the correlators in the following equations do not have a $q$ or $g$ superscript, the same relation is valid for both the quark-quark and gluon-gluon correlator. Transverse superscripts separated by a slash denote multiple possible values to be considered in sequence between the left- and right-hand side.
} 
(iv) Finally, $\mathcal{P}_{\perp} \mathcal{T}_{\perp}$ combined implies

$$
\begin{aligned}
V_{\lambda^{\prime} \lambda}(\Delta, P, n) & =(-1)^{\lambda^{\prime}-\lambda} V_{-\lambda-\lambda^{\prime}}(-\Delta, P, n), \\
A_{\lambda^{\prime} \lambda}(\Delta, P, n) & =(-1)^{\lambda^{\prime}-\lambda+1} A_{-\lambda-\lambda^{\prime}}(-\Delta, P, n), \\
T_{\lambda^{\prime} \lambda}^{q R / L}(\Delta, P, n) & =(-1)^{\lambda^{\prime}-\lambda+1} T_{-\lambda-\lambda^{\prime}}^{q R / L}(-\Delta, P, n), \\
T_{\lambda^{\prime} \lambda}^{g R R / L L}(\Delta, P, n) & =(-1)^{\lambda^{\prime}-\lambda} T_{-\lambda-\lambda^{\prime}}^{g R R / L L}(-\Delta, P, n),
\end{aligned}
$$

where the notation $\tilde{P}$ is defined in Eq. (A3).

\section{Leading twist-2 quark transversity GPDs}

The leading twist- 2 transversity quark GPDs are chiral odd and defined by matrix elements of the tensor correlator $T_{\lambda^{\prime} \lambda}^{q i}$. They are scalar functions depending on Lorentz invariants $x, \xi, t$ multiplying all possible independent tensor structures that appear in the decomposition of the correlator matrix element. These tensor structures are built from the available four-vectors $\epsilon, \epsilon^{\prime}, n, P, \Delta$, and the decomposition has to obey the symmetry constraints given in the previous section. We decompose the correlator as

$$
\begin{aligned}
\int \frac{d \kappa}{2 \pi} e^{2 i x \kappa(P n)}\left\langle p^{\prime} \lambda^{\prime}\left|\bar{\psi}(-\kappa n)\left(i n_{\mu} \sigma^{\mu i}\right) \psi(\kappa n)\right| p \lambda\right\rangle= & M \frac{\left(\epsilon^{\prime *} n\right) \epsilon^{i}-\epsilon^{\prime * i}(\epsilon n)}{2 \sqrt{2}(P n)} H_{1}^{q T}(x, \xi, t) \\
& +M\left[\frac{2 P^{i}(\epsilon n)\left(\epsilon^{\prime *} n\right)}{2 \sqrt{2}(P n)^{2}}-\frac{(\epsilon n) \epsilon^{\prime i *}+\epsilon^{i}\left(\epsilon^{\prime *} n\right)}{2 \sqrt{2}(P n)}\right] H_{2}^{q T}(x, \xi, t) \\
& +\left[\frac{\left(\epsilon^{\prime *} n\right) \Delta^{i}-\epsilon^{\prime i *}(\Delta n)}{M(P n)}(\epsilon P)-\frac{(\epsilon n) \Delta^{i}-\epsilon^{i}(\Delta n)}{M(P n)}\left(\epsilon^{\prime *} P\right)\right] H_{3}^{q T}(x, \xi, t) \\
& +\left[\frac{\left(\epsilon^{\prime *} n\right) \Delta^{i}-\epsilon^{\prime i *}(\Delta n)}{M(P n)}(\epsilon P)+\frac{(\epsilon n) \Delta^{i}-\epsilon^{i}(\Delta n)}{M(P n)}\left(\epsilon^{\prime *} P\right)\right] H_{4}^{q T}(x, \xi, t) \\
& +M\left[\frac{\left(\epsilon^{\prime *} n\right) \Delta^{i}-\epsilon^{\prime i *}(\Delta n)}{2 \sqrt{2}(P n)^{2}}(\epsilon n)+\frac{(\epsilon n) \Delta^{i}-\epsilon^{i}(\Delta n)}{2 \sqrt{2}(P n)^{2}}\left(\epsilon^{\prime *} n\right)\right] H_{5}^{q T}(x, \xi, t) \\
& +\frac{\left(\Delta^{i}+2 \xi P^{i}\right)}{M}\left(\epsilon^{\prime *} \epsilon\right) H_{6}^{q T}(x, \xi, t)+\frac{\left(\Delta^{i}+2 \xi P^{i}\right)}{M} \frac{\left(\epsilon^{\prime *} P\right)(\epsilon P)}{M^{2}} H_{7}^{q T}(x, \xi, t) \\
& +\left[\frac{\left(\epsilon^{\prime *} n\right) P^{i}-\epsilon^{\prime i *}(P n)}{M(P n)}(\epsilon P)+\frac{(\epsilon n) P^{i}-\epsilon^{i}(P n)}{M(P n)}\left(\epsilon^{\prime *} P\right)\right] H_{8}^{q T}(x, \xi, t) \\
& +\left[\frac{\left(\epsilon^{\prime *} n\right) P^{i}-\epsilon^{\prime i *}(P n)}{M(P n)}(\epsilon P)-\frac{(\epsilon n) P^{i}-\epsilon^{i}(P n)}{M(P n)}\left(\epsilon^{\prime *} P\right)\right] H_{9}^{q T}(x, \xi, t) .
\end{aligned}
$$

All nine tensor structures are linearly independent, consequently so are the nine GPDs. This can be best seen by considering the transformation between the GPDs and helicity amplitudes (see Appendix C). Using the Hermiticity, parity and time reversal constraints on the correlators written down in Sec. II B, we find the following properties of the GPDs:

(i) All nine $H_{i}^{q T}$ are real.

(ii) Even/odd behavior in skewness $\xi$,

$$
\begin{aligned}
& H_{i}^{q T}(x,-\xi, t)=H_{i}^{q T}(x, \xi, t) \quad i \in\{1,4,5,6,7,9\}, \\
& H_{i}^{q T}(x,-\xi, t)=-H_{i}^{q T}(x, \xi, t) \quad i \in\{2,3,8\} .
\end{aligned}
$$

(iii) Sum rules and form factors of local currents: due to the odd nature of the GPD or the presence of $n^{\mu} n^{\nu} /(P n)^{2}$ in the accompanying tensor, we have the following sum rules that equal zero:

$$
\int_{-1}^{1} \mathrm{~d} x H_{i}^{q T}(x, \xi, t)=0 \quad i \in\{2,3,5,8\} .
$$

The first moments of the other five GPDs give form factors of local tensor currents.

(iv) Forward limit: this corresponds to $\Delta=0, \xi=0$, $(\epsilon P)=\left(\epsilon^{\prime *} P\right)=0$. The only GPD that does not decouple and is nonzero in this limit is $H_{1}^{q T}(x, 0,0)$. It can be connected to the collinear parton distribution function (pdf) $h_{1}(x)$ defined in Refs. [20,21],

$$
h_{1}(x)=H_{1}^{q T}(x, 0,0) .
$$

The correlators of Eq. (9) can be connected to partonhadron scattering amplitudes in $u$-channel kinematics. We can thus write the helicity amplitudes of quark-hadron scattering $\mathcal{A}_{\lambda^{\prime} \mu^{\prime} ; \lambda \mu}^{q}$ [with $\mu\left(\mu^{\prime}\right)$ the light-front helicity of the outgoing (incoming) parton line] as certain projections of Eq. (9), and one has for the chiral odd helicity amplitudes [19],

$$
\mathcal{A}_{\lambda^{\prime}+; \lambda-}^{q}=\frac{1}{2} T_{\lambda^{\prime} \lambda}^{q R}, \quad \mathcal{A}_{\lambda^{\prime}-; \lambda+}^{q}=-\frac{1}{2} T_{\lambda^{\prime} \lambda}^{q L}
$$


Plugging the explicit expressions of the polarization four-vectors of Eq. (7) in the decomposition of Eq. (17), we obtain a linear set of transformations between the nine independent helicity amplitudes $\mathcal{A}_{\lambda^{\prime}+; \lambda_{-}}^{q}$ and the nine transversity GPDs $H_{i}^{q T}$. This set of equations and their inverse are listed in Appendix C.

\section{Leading twist-2 gluon transversity GPDs}

The leading twist-2 transversity gluon GPDs are defined by matrix elements of the tensor correlator $T_{\lambda^{\prime} \lambda}^{g i j}$. We decompose this correlator as

$$
\begin{aligned}
- & \frac{2}{(P n)} \int \frac{d \kappa}{2 \pi} e^{2 i x k(P n)}\left\langle p^{\prime} \lambda^{\prime}\left|\operatorname{Tr} \hat{\boldsymbol{S}}\left[n_{\alpha} G^{\alpha i}(-\kappa n) n_{\beta} G^{\beta j}(\kappa n)\right]\right| p \lambda\right\rangle \\
= & \hat{\boldsymbol{S}}\left\{\left(\Delta^{i}+2 \xi P^{i}\right) \frac{\left(\epsilon^{\prime *} n\right) \epsilon^{j}-\epsilon^{\prime j *}(\epsilon n)}{(P n)} H_{1}^{g T}(x, \xi, t)+\left(\Delta^{i}+2 \xi P^{i}\right)\left[\frac{2 P^{j}(\epsilon n)\left(\epsilon^{\prime *} n\right)}{(P n)^{2}}-\frac{\left.(\epsilon n) \epsilon^{\prime j *}+\epsilon^{j}\left(\epsilon^{\prime *} n\right)\right]}{(P n)}\right] H_{2}^{g T}(x, \xi, t)\right. \\
& +\frac{\left(\Delta^{i}+2 \xi P^{i}\right)}{M}\left[\frac{\left(\epsilon^{\prime *} n\right) \Delta^{j}-\epsilon^{\prime j *}(\Delta n)}{M(P n)}(\epsilon P)-\frac{(\epsilon n) \Delta^{j}-\epsilon^{j}(\Delta n)}{M(P n)}\left(\epsilon^{\prime *} P\right)\right] H_{3}^{g T}(x, \xi, t) \\
& +\frac{\left(\Delta^{i}+2 \xi P^{i}\right)}{M}\left[\frac{\left(\epsilon^{\prime *} n\right) \Delta^{j}-\epsilon^{\prime j *}(\Delta n)}{M(P n)}(\epsilon P)+\frac{(\epsilon n) \Delta^{j}-\epsilon^{j}(\Delta n)}{M(P n)}\left(\epsilon^{\prime *} P\right)\right] H_{4}^{g T}(x, \xi, t) \\
& -\left[\frac{\left(\epsilon^{\prime *} n\right) P^{i}-(P n) \epsilon^{\prime i *}}{(P n)}\right]\left[\frac{(\epsilon n) P^{j}-(P n) \epsilon^{j}}{(P n)}\right] H_{5}^{g T}(x, \xi, t) \\
& +\left[\frac{\left.\left(\epsilon^{\prime *} n\right) \Delta^{i}-(\Delta n) \epsilon^{\prime i *}\right]}{2(P n)}\right]\left[\frac{(\epsilon n) \Delta^{j}-(\Delta n) \epsilon^{j}}{2(P n)}\right] H_{6}^{g T}(x, \xi, t)+\frac{\left(\Delta^{i}+2 \xi P^{i}\right)}{M} \frac{\left(\Delta^{j}+2 \xi P^{j}\right)}{M} \frac{\left(\epsilon^{\prime *} P\right)(\epsilon P)}{M^{2}} H_{7}^{g T}(x, \xi, t) \\
& +\frac{\Delta^{i}+2 \xi P^{i}}{M}\left[\frac{\left(\epsilon^{\prime *} n\right) P^{j}-\epsilon^{\prime j *}(P n)}{M(P n)}(\epsilon P)+\frac{(\epsilon n) P^{j}-\epsilon^{j}(P n)}{M(P n)}\left(\epsilon^{\prime *} P\right)\right] H_{8}^{g T}(x, \xi, t) \\
& \left.+\frac{\Delta^{i}+2 \xi P^{i}}{M}\left[\frac{\left(\epsilon^{\prime *} n\right) P^{j}-\epsilon^{\prime j *}(P n)}{M(P n)}(\epsilon P)-\frac{(\epsilon n) P^{j}-\epsilon^{j}(P n)}{M(P n)}\left(\epsilon^{\prime *} P\right)\right] H_{9}^{g T}(x, \xi, t)\right\}
\end{aligned}
$$

The tensor structures that appear in the above equation are linearly independent. This is again best observed from the relations between the transversity GPDs and helicity amplitudes written out in Appendix C.

Using the Hermiticity, parity and time reversal constraints on the correlators written down in Sec. II B, we find the following properties of the GPDs:

(i) All nine GPDs are real and even in $x$.

(ii) Similarly as for the quark GPDs, the even or odd behavior in skewness $\xi$ is as follows:

$$
\begin{aligned}
& H_{i}^{g T}(x,-\xi, t)=H_{i}^{g T}(x, \xi, t) \quad i \in\{1,4,5,6,7,9\}, \\
& H_{i}^{g T}(x,-\xi, t)=-H_{i}^{g T}(x, \xi, t) \quad i \in\{2,3,8\} .
\end{aligned}
$$

(iii) Sum rules and form factors of local currents: due to the odd nature of the GPD, we have the following sum rules that equal zero:

$$
\int_{-1}^{1} \mathrm{~d} x H_{i}^{g T}(x, \xi, t)=0 \quad i \in\{2,3,8\},
$$

the first moments of the remaining 6 GPDs give form factors of local tensor currents.

(iv) Forward limit: the only GPD that does not decouple and is nonzero is $H_{5}^{g T}(x, 0,0)$. It can be connected to structure function $x \Delta$ defined in Ref. [22] [Eq. (1) within] or the collinear pdf $x h_{1 T T}(x)$ in Ref. [23] [Eq. (2.38) within],

$$
H_{5}^{g T}(x, 0,0)=x h_{1 T T}(x) .
$$

This pdf is unique to the spin- 1 case as a spin $1 / 2$ hadron cannot compensate the gluon helicity flip.

The relation between helicity flip gluon-hadron helicity amplitudes $\mathcal{A}_{\lambda^{\prime}+; \lambda-}^{g}$ and the correlators of Eq. (10) is given by [19]

$$
\mathcal{A}_{\lambda^{\prime}+; \lambda-}^{g}=\frac{1}{2} T_{\lambda^{\prime} \lambda}^{g R R}, \quad \mathcal{A}_{\lambda^{\prime}-; \lambda+}^{g}=\frac{1}{2} T_{\lambda^{\prime} \lambda}^{g L L} .
$$

As for the quark sector, we can plug in the explicit expressions for the spin-1 polarization four-vectors and obtain the transformation equations between the helicity amplitudes and the gluon transversity GPDs listed in Appendix C.

\section{DEUTERON CONVOLUTION MODEL: FORMALISM}

In this section, we derive the expression of the spin 1 transversity GPDs for the case of the deuteron in the impulse approximation (IA). In the IA, we consider the 


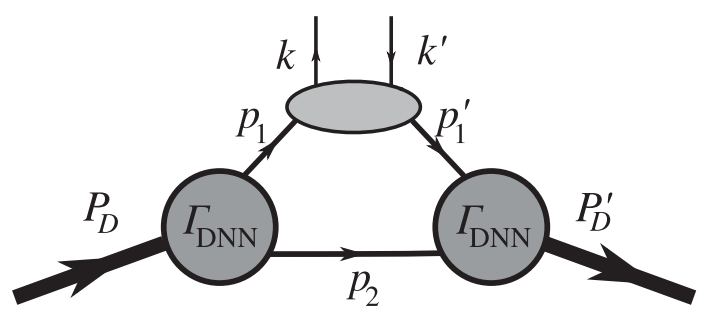

FIG. 2. Diagrammatic depiction of the impulse approximation for the deuteron GPDs, considering the $N N$ component of the deuteron, for kinematics where $x>\xi$.

dominant $N N$ component of the deuteron depicted in the diagram of Fig. 2. The two quark lines in the correlators of Eq. (9) are attached to the same nucleon, and the second nucleon acts as a "spectator". This is a standard first order approximation in the computation of partonic properties of nuclei [24-29]. The derivation presented here follows the approach used in Ref. [16]: the correlator $T_{\lambda^{\prime} \lambda}^{q R / L}$ for the deuteron is expressed as a convolution of the deuteron light-front wave function with similar correlators for the nucleon. The latter are expressed through the four transversity GPDs of the nucleon. In the final step the correlators can be connected to the transversity deuteron GPDs by inverting the relations between the complete set of helicity amplitudes defined by Eqs. (C1)-(C9) and the transversity spin 1 GPDs.

\section{A. Kinematics and conventions}

As we will be dealing with kinematic variables on both the nuclear and nucleon level, we amend the notations of Sec. II A to differentiate clearly between the two. Using the four-momenta shown in Fig. 2, we introduce the following kinematic variables:

$$
\begin{aligned}
\bar{P}_{D} & =\frac{1}{2}\left(P_{D}+P_{D}^{\prime}\right), \quad \bar{p}_{1}=\frac{1}{2}\left(p_{1}+p_{1}^{\prime}\right), \\
\Delta & =P_{D}^{\prime}-P_{D}=p_{1}^{\prime}-p_{1}, \\
\xi & =-\frac{(\Delta n)}{2\left(\bar{P}_{D} n\right)}, \quad \xi_{N}=-\frac{(\Delta n)}{2\left(\bar{p}_{1} n\right)}, \\
\bar{k} & =\frac{1}{2}\left(k+k^{\prime}\right), \quad x=\frac{(\bar{k} n)}{\left(\bar{P}_{D} n\right)}, \quad x_{N}=\frac{(\bar{k} n)}{\left(\bar{p}_{1} n\right)} .
\end{aligned}
$$

We introduce light-front momentum fractions for the nucleons,

$$
\begin{array}{ll}
\alpha_{1}=2 \frac{p_{1} n}{P_{D} n}, & \alpha_{2}=2 \frac{p_{2} n}{P_{D} n}=2-\alpha_{1}, \\
\alpha_{1}^{\prime}=2 \frac{p_{1}^{\prime} n}{P_{D}^{\prime} n}, & \alpha_{2}^{\prime}=2 \frac{p_{2} n}{P_{D}^{\prime} n}=2-\alpha_{1}^{\prime},
\end{array}
$$

and we have the following useful identities:

$$
\begin{aligned}
\frac{\left(p_{1} n\right)}{\left(\bar{P}_{D} n\right)} & =\frac{\alpha_{1}(1+\xi)}{2}, \quad \frac{\left(p_{1}^{\prime} n\right)}{\left(\bar{P}_{D} n\right)}=\frac{\alpha_{1}^{\prime}(1-\xi)}{2}, \\
\alpha_{1}(1+\xi) & =\alpha_{1}^{\prime}(1-\xi)+4 \xi, \\
\xi_{N} & =\frac{\xi}{\frac{\alpha_{1}}{2}(1+\xi)-\xi}, \quad x_{N}=\frac{x}{\frac{\alpha_{1}}{2}(1+\xi)-\xi} .
\end{aligned}
$$

The deuteron light-front wave function depends on the following dynamical variable, the three-momentum $\boldsymbol{k}_{d}$ defined by:

$$
\begin{aligned}
\frac{k_{d}^{z}}{E_{k}} & =\alpha_{1}-1, \quad \boldsymbol{k}_{d}^{\perp}=\boldsymbol{p}_{1}^{\perp}-\frac{\alpha_{1}}{2} \boldsymbol{P}_{D}^{\perp}, \\
E_{k_{d}}^{2} & =\boldsymbol{k}_{d}^{2}+m^{2}=\frac{m^{2}+\left(\boldsymbol{k}_{d}^{\perp}\right)^{2}}{\alpha_{1} \alpha_{2}},
\end{aligned}
$$

where $m$ is the nucleon mass. The momentum $\boldsymbol{k}_{d}$ corresponds to the relative momentum of the two on shell nucleons in the light-front boosted deuteron rest frame [30,31]. The first two equations follow from the properties of light-front boosts while the third equation can be obtained by equating $\left(k_{p}+k_{n}\right)^{2}=4 E_{k_{d}}^{2}=2 m^{2}+2\left(k_{n} k_{p}\right)$, where $k_{p}, k_{n}$ are the on shell nucleon momenta of the intermediate $N N$ state.

Finally, the phase space element of the active nucleon can be written as

$d \Gamma_{1}=\frac{d p_{1}^{+} d \boldsymbol{p}_{1}^{\perp}}{(2 \pi)^{3} 2 p_{1}^{+}}=\frac{d \alpha_{1} d \boldsymbol{p}^{\perp}}{(2 \pi)^{3} 2 \alpha_{1}}=\left(2-\alpha_{1}\right) \frac{d^{3} \boldsymbol{k}_{d}}{(2 \pi)^{3} 2 E_{k_{d}}}$.

\section{B. Deuteron light-front wave function}

The deuteron light-front wave function [30-33] is given by the overlap of the deuteron single-particle state with the on shell two-nucleon state, where all states are quantized on the light-front,

$$
\begin{aligned}
& \left\langle N\left(p_{1}, \sigma_{1}\right) ; N\left(p_{2}, \sigma_{2}\right) \mid D\left(P_{D}, \lambda\right)\right\rangle \\
& \equiv(2 \pi)^{\frac{9}{2}} 2 P_{D}^{+} \delta\left(p_{1}^{+}+p_{2}^{+}-P_{D}^{+}\right) \delta\left(\boldsymbol{p}_{1}^{\perp}+\boldsymbol{p}_{2}^{\perp}-\boldsymbol{P}_{D}^{\perp}\right) \\
& \times \Psi_{\lambda}^{D}\left(\boldsymbol{k}_{d}, \sigma_{1}, \sigma_{2}\right) \text {. }
\end{aligned}
$$

All involved momenta $\left(P_{D}, p_{1}, p_{2}\right)$ are on their mass shell, which means light-front energy (minus component of momentum) is not conserved in the transition $D \rightarrow N N$. For the free two-nucleon state in the transition matrix element of Eq. (32), an angular momentum decomposition can be performed in the light-front boosted deuteron rest frame in a way very similar to the case of the nonrelativistic deuteron wave function. The relative motion of the two nucleons in the deuteron rest frame can be projected on spherical harmonics, and for the deuteron a radial $S$-wave $(l=0)$ and $D$-wave $(l=2)$ can be coupled to the total spin 
$S=1$ of the two nucleons to obtain total light-front spin $j=1$. The final form of the deuteron light-front wave function defined through Eq. (32) reflects this angular decomposition,

$$
\begin{aligned}
\Psi_{\lambda}^{D}\left(\boldsymbol{k}, \sigma_{1}, \sigma_{2}\right)= & \sqrt{E_{k}} \sum_{\sigma_{1}^{\prime} \sigma_{2}^{\prime}} \mathcal{D}_{\sigma_{1} \sigma_{1}^{\prime}}^{\frac{1}{2}}\left[R_{f c}\left(k_{p}^{\mu} / m\right)\right] \\
& \times \mathcal{D}_{\sigma_{2} \sigma_{2}^{\prime}}^{\frac{1}{2}}\left[R_{f c}\left(k_{n}^{\mu} / m\right)\right] \Phi_{\lambda}^{D}\left(\boldsymbol{k}_{d}, \sigma_{1}^{\prime}, \sigma_{2}^{\prime}\right),
\end{aligned}
$$

with

$$
\begin{aligned}
\Phi_{\lambda}^{D}\left(\boldsymbol{k}_{d}, \sigma_{1}^{\prime}, \sigma_{2}^{\prime}\right)= & \sum_{\substack{l=0,2 \\
\lambda_{l} \lambda_{S}}}\left\langle l \lambda_{l} 1 \lambda_{S} \mid 1 \lambda\right\rangle\left\langle\frac{1}{2} \sigma_{1}^{\prime} \frac{1}{2} \sigma_{2}^{\prime} \mid 1 \lambda_{S}\right\rangle \\
& \times Y_{l}^{\lambda_{l}}\left(\Omega_{\boldsymbol{k}_{d}}\right) \phi_{l}(k),
\end{aligned}
$$

where the $\phi_{l}(k)$ denote the radial components of the wave function and $Y_{l}^{\lambda_{l}}\left(\Omega_{\boldsymbol{k}}\right)$ are the spherical harmonics.

The deuteron light-front wave function has two different features compared to the nonrelativistic one that deserve highlighting. First, there is the appearance of two Melosh rotations $\mathcal{D}_{\lambda_{i} \lambda_{i}^{l}}^{\frac{1}{2}}\left[R_{f c}\left(k_{i}^{\mu} / m\right)\right]$ [34] in Eq. (33) that encode relativistic spin effects arising from the quantization of particle states (and spin) on the light-front. Second, the dynamical variable that appears in the light-front wave function is the three-momentum $\boldsymbol{k}$. In the calculations presented in this article the radial wave functions $\phi_{l}(k)$ are identified with those from nonrelativistic wave function parametrizations. We want to stress that this does not correspond with approximating the light-front wave function with the nonrelativistic one given the differences pointed out above. This approach can be justified for momenta up to a few $100 \mathrm{MeV}$ given the small binding energy of the deuteron. In Ref. [35], an explicit comparison between the instant-form and front-form wave function for a two-particle bound state was carried out in a toy model. The connection between the nonrelativistic instant form and light-front wave function as in Eq. (33) was found to hold for $\epsilon_{B} / M_{D}<0.002$ (with $\epsilon_{B}, M_{D}$ the deuteron binding energy and mass), which holds for the deuteron case.

\section{Nucleon chiral odd quark GPDs}

As the tensor correlator defining the nucleon chiral odd quark GPDs appears in the IA derivation, we briefly summarize expressions for these in this section. We use the standard parametrization for the nucleon chiral odd quark GPDs introduced in Ref. [19],

$$
\begin{aligned}
\int \frac{d \kappa}{2 \pi} e^{2 i x_{N} \kappa\left(\bar{p}_{1} n\right)}\left\langle p_{1}^{\prime} \sigma_{1}^{\prime}\left|\bar{\psi}(-\kappa n)\left(i n_{\mu} \sigma^{\mu i}\right) \psi(\kappa n)\right| p_{1} \sigma_{1}\right\rangle \\
=\frac{1}{2\left(\bar{p}_{1} n\right)} \bar{u}\left(p_{1}^{\prime}, \sigma_{1}^{\prime}\right)\left[H_{T}^{q}\left(i n_{\mu} \sigma^{\mu i}\right)+\tilde{H}_{T}^{q} \frac{\left(\bar{p}_{1} n\right) \Delta^{i}-(\Delta n) \bar{p}_{1}^{i}}{m^{2}}\right. \\
\left.\quad+E_{T}^{q} \frac{(\gamma n) \Delta^{i}-(\Delta n) \gamma^{i}}{2 m}+\tilde{E}_{T}^{q} \frac{(\gamma n) \bar{p}_{1}^{i}-\left(\bar{p}_{1} n\right) \gamma^{i}}{m}\right] u\left(p_{1}, \sigma_{1}\right) .
\end{aligned}
$$

Substituting the standard light-front spinors [36], we list explicit expressions for the spinor bilinears multiplying the GPDs in the above expression. For \pm and $\mp$ appearing in the following expressions the upper sign comes with the $R$ component, the lower one with the $L$ component. We have

$$
\begin{aligned}
& \frac{1}{2\left(\bar{p}_{1} n\right)} \bar{u}\left(p_{1}^{\prime}, \sigma_{1}^{\prime}\right)\left(i n_{\mu} \sigma^{\mu R / L}\right) u\left(p_{1}, \sigma_{1}\right) \\
& =-\delta_{-\sigma_{1}^{\prime}, \sigma_{1}}\left(2 \sigma_{1} \mp 1\right) \sqrt{1-\xi_{N}^{2}}, \\
& \frac{1}{2\left(\bar{p}_{1} n\right)} \bar{u}\left(p_{1}^{\prime}, \sigma_{1}^{\prime}\right)\left[\frac{\left(\bar{p}_{1} n\right) \Delta^{R / L}-(\Delta n) \bar{p}_{1}^{R / L}}{m^{2}}\right] u\left(p_{1}, \sigma_{1}\right) \\
& =\delta_{\sigma_{1}^{\prime}, \sigma} \frac{\sqrt{t_{0 N}-t}}{m} e^{ \pm i \phi_{1}} \\
& \quad-\delta_{-\sigma_{1}^{\prime}, \sigma_{1}} \sigma_{1} \frac{\sqrt{1-\xi_{N}^{2}}\left(t_{0 N}-t\right)}{m^{2}} e^{\left(2 \sigma_{1} \pm 1\right) i \phi_{1}}, \\
& \frac{1}{2\left(\bar{p}_{1} n\right)} \bar{u}\left(p_{1}^{\prime}, \sigma_{1}^{\prime}\right)\left[\frac{(\gamma n) \Delta^{R / L}-(\Delta n) \gamma^{R / L}}{2 m}\right] u\left(p_{1}, \sigma_{1}\right) \\
& =\delta_{\sigma_{1}^{\prime}, \sigma_{1}} \frac{\left(1 \mp 2 \sigma_{1} \xi_{N}\right) \sqrt{t_{0 N}-t}}{2 m} e^{ \pm i \phi_{1}} \\
& \quad+\delta_{-\sigma_{1}^{\prime}, \sigma_{1}}\left(2 \sigma_{1} \mp 1\right) \frac{\xi_{N}^{2}}{\sqrt{1-\xi_{N}^{2}}}, \\
& \frac{1}{2\left(\bar{p}_{1} n\right)} \bar{u}\left(p_{1}^{\prime}, \sigma_{1}^{\prime}\right)\left[\frac{(\gamma n) \bar{p}_{1}^{R / L}-\left(\bar{p}_{1} n\right) \gamma^{R / L}}{m}\right] u\left(p_{1}, \sigma_{1}\right) \\
& = \pm \delta_{\sigma_{1}^{\prime}, \sigma_{1}} 2 \sigma_{1} \frac{\left(1 \mp 2 \sigma_{1} \xi_{N}\right) \sqrt{t_{0 N}-t}}{2 m} e^{ \pm i \phi_{1}} \\
& -\delta_{-\sigma_{1}^{\prime}, \sigma_{1}}\left(2 \sigma_{1} \mp 1\right) \frac{\xi_{N}}{\sqrt{1-\xi_{N}^{2}}},
\end{aligned}
$$

where $\phi_{1}$ is the azimuthal angle of the four-vector $\Delta+2 \xi_{N} \bar{p}_{1}$ and

$$
t_{0 N}=-\frac{4 m^{2} \xi_{N}^{2}}{1-\xi_{N}^{2}}
$$

\section{Impulse approximation derivation}

As the following derivation does not depend on the exact operator in the correlator, we leave it unspecified and call it $\hat{A}$. Consequently the equations below apply to any quarkquark or gluon-gluon GPD correlator written down in Sec. II B. 
We start by inserting two complete sets of on shell two-nucleon states in the correlator, use Eq. (32) to introduce the deuteron light-front wave functions and Eq. (A6) to evaluate the integrations over the spectator nucleon phase space elements,

$$
\begin{aligned}
& \int \frac{d \kappa}{2 \pi} e^{2 i x \kappa\left(\bar{P}_{D} n\right)}\left\langle P_{D}^{\prime} \lambda^{\prime}|\hat{A}| P_{D}, \lambda\right\rangle=\sum_{N} \int \frac{d p_{1}^{+} d p_{1}^{\perp}}{2 p_{1}^{+}} \frac{d p_{1}^{\prime+} d \boldsymbol{p}_{1}^{\prime \perp}}{2 p_{1}^{\prime+}} \frac{d p_{2}^{+} d p_{2}^{\perp}}{2 p_{2}^{+}} 2 P_{D}^{+} 2 P_{D}^{\prime+} \delta^{+\perp}\left(P_{D}^{\prime}-p_{1}^{\prime}-p_{2}\right) \\
& \times \delta^{+\perp}\left(P_{D}-p_{1}-p_{2}\right) \Theta\left(\frac{\alpha_{1}}{2}(1+\xi)-|x|-\xi\right) \\
& \times\left[\Theta(\xi) \Theta\left(\frac{\alpha_{1}}{2}(1+\xi)-2 \xi\right)+\Theta(-\xi) \Theta\left(\frac{\alpha_{1}}{2}(1+\xi)\right)\right] \\
& \times \sum_{\sigma_{1} \sigma_{1}^{\prime} \sigma_{2}} \Psi_{\lambda^{\prime}}^{* D}\left(\boldsymbol{k}_{d}^{\prime}, \sigma_{1}^{\prime}, \sigma_{2}\right) \Psi_{\lambda}^{D}\left(\boldsymbol{k}_{d}, \sigma_{1}, \sigma_{2}\right) \int \frac{d \kappa}{2 \pi} e^{2 i x_{N} \kappa\left(\bar{p}_{1} n\right)}\left\langle p_{1}^{\prime} \sigma_{1}^{\prime}|\hat{A}| p_{1} \sigma_{1}\right\rangle \\
& =\sum_{N} \int \frac{d \alpha_{1} d \boldsymbol{p}_{1}^{\perp}}{\alpha_{1}} \frac{d \alpha_{1}^{\prime} d \boldsymbol{p}_{1}^{\prime}}{\alpha_{1}^{\prime}} \frac{P_{D}^{+} P_{D}^{\prime+}}{2 p_{2}^{+}} \delta\left(-\Delta^{+}-p_{1}^{+}+p_{1}^{\prime+}\right) \delta\left(-\boldsymbol{\Delta}^{\perp}-\boldsymbol{p}_{1}^{\perp}+\boldsymbol{p}_{1}^{\prime}{ }_{1}\right) \\
& \times \Theta\left(\frac{\alpha_{1}}{2}(1+\xi)-|x|-\xi\right)\left[\Theta(\xi) \Theta\left(\frac{\alpha_{1}}{2}(1+\xi)-2 \xi\right)+\Theta(-\xi) \Theta\left(\frac{\alpha_{1}}{2}(1+\xi)\right)\right] \\
& \times \sum_{\sigma_{1} \sigma_{1}^{\prime} \sigma_{2}} \Psi_{\lambda^{\prime}}^{* D}\left(\boldsymbol{k}_{d}^{\prime}, \sigma_{1}^{\prime}, \sigma_{2}\right) \Psi_{\lambda}^{D}\left(\boldsymbol{k}_{d}, \sigma_{1}, \sigma_{2}\right) \int \frac{d \kappa}{2 \pi} e^{2 i x_{N} \kappa\left(\bar{p}_{1} n\right)}\left\langle p_{1}^{\prime} \sigma_{1}^{\prime}|\hat{A}| p_{1} \sigma_{1}\right\rangle \\
& =\sum_{N} \int \frac{d \alpha_{1} d \boldsymbol{p}_{1}^{\perp}}{\alpha_{1}} \frac{d \alpha_{1}^{\prime} d \boldsymbol{p}_{1}^{\prime}}{\alpha_{1}^{\prime}} \frac{2}{2-\alpha_{1}} \delta\left(\alpha_{1}^{\prime}-\alpha_{1} \frac{1+\xi}{1-\xi}+4 \frac{\xi}{1-\xi}\right) \delta\left(-\boldsymbol{\Delta}^{\perp}-\boldsymbol{p}_{1}^{\perp}+\boldsymbol{p}_{1}^{\prime}\right) \\
& \times \Theta\left(\frac{\alpha_{1}}{2}(1+\xi)-|x|-\xi\right)\left[\Theta(\xi) \Theta\left(\frac{\alpha_{1}}{2}(1+\xi)-2 \xi\right)+\Theta(-\xi) \Theta\left(\frac{\alpha_{1}}{2}(1+\xi)\right)\right] \\
& \times \sum_{\sigma_{1} \sigma_{1}^{\prime} \sigma_{2}} \Psi_{\lambda^{\prime}}^{* D}\left(\boldsymbol{k}_{d}^{\prime}, \sigma_{1}^{\prime}, \sigma_{2}\right) \Psi_{\lambda}^{D}\left(\boldsymbol{k}_{d}, \sigma_{1}, \sigma_{2}\right) \int \frac{d \kappa}{2 \pi} e^{2 i x_{N} \kappa\left(\bar{p}_{1} n\right)}\left\langle p_{1}^{\prime} \sigma_{1}^{\prime}|\hat{A}| p_{1} \sigma_{1}\right\rangle \\
& =2 \sum_{N} \int \frac{d \alpha_{1} d \boldsymbol{k}_{d}^{\perp}}{\alpha_{1}\left(2-\alpha_{1}\right)} \frac{d \alpha_{1}^{\prime} d \boldsymbol{k}^{\prime} \frac{\perp}{d}}{\alpha_{1}^{\prime}} \delta\left(\alpha_{1}^{\prime}-\alpha_{1} \frac{1+\xi}{1-\xi}+4 \frac{\xi}{1-\xi}\right) \\
& \times \delta\left(\boldsymbol{k}^{\prime} \frac{\perp}{d}-\boldsymbol{k}_{d}^{\perp}-\frac{1-\frac{\alpha_{1}}{2}}{1-\xi} \Delta^{\perp}-2 \xi \frac{1-\frac{\alpha_{1}}{2}}{1-\xi} \overline{\boldsymbol{P}}_{D}^{\perp}\right) \Theta\left(\frac{\alpha_{1}}{2}(1+\xi)-|x|-\xi\right) \\
& \times\left[\Theta(\xi) \Theta\left(\frac{\alpha_{1}}{2}(1+\xi)-2 \xi\right)+\Theta(-\xi) \Theta\left(\frac{\alpha_{1}}{2}(1+\xi)\right)\right] \\
& \times \sum_{\sigma_{1} \sigma_{1}^{\prime} \sigma_{2}} \Psi_{\lambda^{\prime}}^{* D}\left(\boldsymbol{k}_{d}^{\prime}, \sigma_{1}^{\prime}, \sigma_{2}\right) \Psi_{\lambda}^{D}\left(\boldsymbol{k}_{d}, \sigma_{1}, \sigma_{2}\right) \int \frac{d \kappa}{2 \pi} e^{2 i x_{N} \kappa\left(\bar{p}_{1} n\right)}\left\langle p_{1}^{\prime} \sigma_{1}^{\prime}|\hat{A}| p_{1} \sigma_{1}\right\rangle .
\end{aligned}
$$

In the third step a factor $2 \bar{P}_{D}$ was brought into the Dirac delta function for the plus components. The sum $N$ is over the two possible active nucleons. The Heaviside functions originate from the requirement of positive light-front plus components for the on shell intermediate states. The $|x|>|\xi|$ region gives the first Heaviside, the ERBL region the remaining ones.

Up to here the derivation is valid for any correlator considered in Sec. II B. In the next step, we specialize to the case of the twist-2 chiral odd quark GPDs. By taking $\hat{A}=\bar{\psi}(-\kappa n)\left(i n_{\mu} \sigma^{\mu R / L}\right) \psi(\kappa n)$ in Eq. (38) and using Eqs. (35) and (36) we arrive at 


$$
\begin{aligned}
T_{\lambda^{\prime} \lambda}^{R / L}= & 4 \int \frac{d \alpha_{1} d \boldsymbol{k}_{d}^{\perp}}{\alpha_{1}\left(2-\alpha_{1}\right)} \frac{d \alpha_{1}^{\prime} d \boldsymbol{k}_{d}^{\prime \perp}}{\alpha_{1}^{\prime}} \delta\left(\alpha_{1}^{\prime}-\alpha_{1} \frac{1+\xi}{1-\xi}+4 \frac{\xi}{1-\xi}\right) \delta\left(\boldsymbol{k}_{d}^{\prime \perp}-\boldsymbol{k}_{d}^{\perp}-\frac{1-\frac{\alpha_{1}}{2}}{1-\xi} \Delta^{\perp}-2 \xi \frac{1-\frac{\alpha_{1}}{2}}{1-\xi} \overline{\boldsymbol{P}}_{D}^{\perp}\right) \\
& \times \Theta\left(\frac{\alpha_{1}}{2}(1+\xi)-|x|-\xi\right)\left[\Theta(\xi) \Theta\left(\frac{\alpha_{1}}{2}(1+\xi)-2 \xi\right)+\Theta(-\xi) \Theta\left(\frac{\alpha_{1}}{2}(1+\xi)\right)\right] \\
& \times \sum_{\sigma_{1} \sigma_{1}^{\prime} \sigma_{2}} \Psi_{\lambda^{\prime}}^{* D}\left(\boldsymbol{k}_{d}^{\prime}, \sigma_{1}^{\prime}, \sigma_{2}\right) \Psi_{\lambda}^{D}\left(\boldsymbol{k}_{d}, \sigma_{1}, \sigma_{2}\right) \cdot\left[-\delta_{-\sigma_{1}^{\prime}, \sigma_{1}}\left(2 \sigma_{1} \mp 1\right) \sqrt{1-\xi_{N}^{2}} H_{T}^{\mathrm{IS}}\left(x_{N}, \xi_{N}, t\right)\right. \\
& +\left(\delta_{\sigma_{1}^{\prime}, \sigma_{1}} \frac{\sqrt{t_{0}-t}}{m} e^{ \pm i \phi_{1}}-\delta_{-\sigma_{1}^{\prime}, \sigma_{1}} \sigma_{1} \frac{\sqrt{1-\xi_{N}^{2}}\left(t_{0}-t\right)}{m^{2}} e^{\left(2 \sigma_{1} \pm 1\right) i \phi_{1}}\right) \tilde{H}_{T}^{\mathrm{IS}}\left(x_{N}, \xi_{N}, t\right) \\
& +\left(\delta_{\sigma_{1}^{\prime}, \sigma_{1}} \frac{\left(1 \mp 2 \sigma_{1} \xi_{N}\right) \sqrt{t_{0}-t}}{2 m} e^{ \pm i \phi_{1}}+\delta_{-\sigma_{1}^{\prime}, \sigma_{1}}\left(2 \sigma_{1} \mp 1\right) \frac{\xi_{N}^{2}}{\sqrt{1-\xi_{N}^{2}}}\right) E_{T}^{\mathrm{IS}}\left(x_{N}, \xi_{N}, t\right) \\
& \left.+\left( \pm \delta_{\sigma_{1}^{\prime}, \sigma_{1}} 2 \sigma_{1} \frac{\left(1 \mp 2 \sigma_{1} \xi_{N}\right) \sqrt{t_{0}-t}}{2 m} e^{ \pm i \phi_{1}}-\delta_{-\sigma_{1}^{\prime}, \sigma_{1}}\left(2 \sigma_{1} \mp 1\right) \frac{\xi_{N}}{\sqrt{1-\xi_{N}^{2}}}\right) \tilde{E}_{T}^{\mathrm{IS}}\left(x_{N}, \xi_{N}, t\right)\right],
\end{aligned}
$$

where the nucleon GPDs are the isoscalar combinations

$X^{\mathrm{IS}}\left(x_{N}, \xi_{N}, t\right)=\frac{1}{2}\left(X^{u}\left(x_{N}, \xi_{N}, t\right)+X^{d}\left(x_{N}, \xi_{N}, t\right)\right)$,

originating from the isoscalar nature of the deuteron $n p$ component considered here. Because of the nonconservation of the minus component in the $D \rightarrow N N$ vertex, the $t$ appearing in the nucleon GPDs is in principle different from the $t$ defined in the beginning [i.e., for the deuteron as defined in Eq. (2)]. Due to the small binding energy $\epsilon_{B}$ of the deuteron, the difference between the two will go as $\epsilon_{B}$ over some larger scale and can be neglected in a first approximation. The deuteron transversity GPDs can be obtained from Eq. (39) by first calculating the helicity amplitudes [Eq. (21)] and subsequently using the results of Appendix C [Eqs. (C21)-(C29)] to compute the GPDs from the helicity amplitudes.

Comparing our derivation with the one presented in Ref. [16], we notice the following differences. Equation numbers mentioned below refer to the ones in Ref. [16]:

(i) Equation (A2) is missing a factor $1 / 2$ in the righthand side so that the deuteron particle state is correctly normalized. As a consequence, Eq. (19) (and following) need an additional factor 1/4.

(ii) Equation (29) should have a prefactor of $\frac{1}{16 \pi^{3}}$. There is a factor of 2 missing in the transition from Eq. (28) to (29) and a factor of $1 / 4$ from the first bullet above.

(iii) The phase of Eq. (31) should read $\eta_{\lambda}=$ $\left(2 \lambda \tilde{\Delta}_{x}+i \tilde{\Delta}_{y}\right) /\left|\tilde{\Delta}_{\perp}\right|$. This can also be inferred from the helicity amplitudes written down in Eq. (61) of Ref. [1], where a factor $e^{ \pm i \phi}$ is written in the $\mathcal{A}_{\mp+, \pm+}$ amplitudes.

\section{DEUTERON CONVOLUTION MODEL: RESULTS}

In this section, we use Eq. (39) in combination with Eq. (21) and Eqs. (C21)-(C29) to compute the helicity amplitudes and transversity GPDs in the quark sector for the deuteron. For the chiral odd nucleon GPDs, we use the parametrization of Goloskokov and Kroll (GK) [37], evaluated at a scale of $\mu=2 \mathrm{GeV}$, and implemented in three different models in Ref. [38] (the figures below use "model 2" therein, which has $\tilde{H}_{T}=H_{T}, E_{T}=\bar{E}_{T}-2 H_{T}$, $\tilde{E}_{T}=0$ ). In the forward limit of this parametrization the helicity pdfs enter [39]. For these we use the parametrization of Ref. [40]. We use the AV18 parametrization of the deuteron wave function [41] unless otherwise noted. As from this section on we are only dealing with quark helicity amplitudes and GPDs, we omit the superscript $q$ for those quantities. We verified that the computed deuteron helicity amplitudes obey all the symmetry constraints listed in Sec. II B, up to the numerical accuracy imposed on the integrations over the active nucleon phase space.

Figures 3 and 4 show the helicity amplitudes and quark transversity GPDs of the deuteron as a function of $x$ (where $-1 \leq x \leq 1)$, in kinematics $\xi=0.1$ and $t=-0.25 \mathrm{GeV}^{2}$. Next to the total result, Figs. 3 and 4 also show the separate contributions to the helicity amplitudes and GPDs when only including the deuteron radial $S$ - or $D$-wave. The remaining difference with the total result originates from $S-D$ interference contributions. For the helicity amplitudes, one observes that the deuteron helicity conserving ones (top row of Fig. 3) are dominated by the pure $S$-wave contribution, whereas the ones with a helicity change for the deuteron receive sizeable contributions from $S-D$ interference terms. The effect of the Melosh rotations is generally smallest in amplitudes dominated by the $S$-wave contribution. Lastly, the two amplitudes with a complete deuteron helicity flip (Fig. 3 bottom row, middle and right 

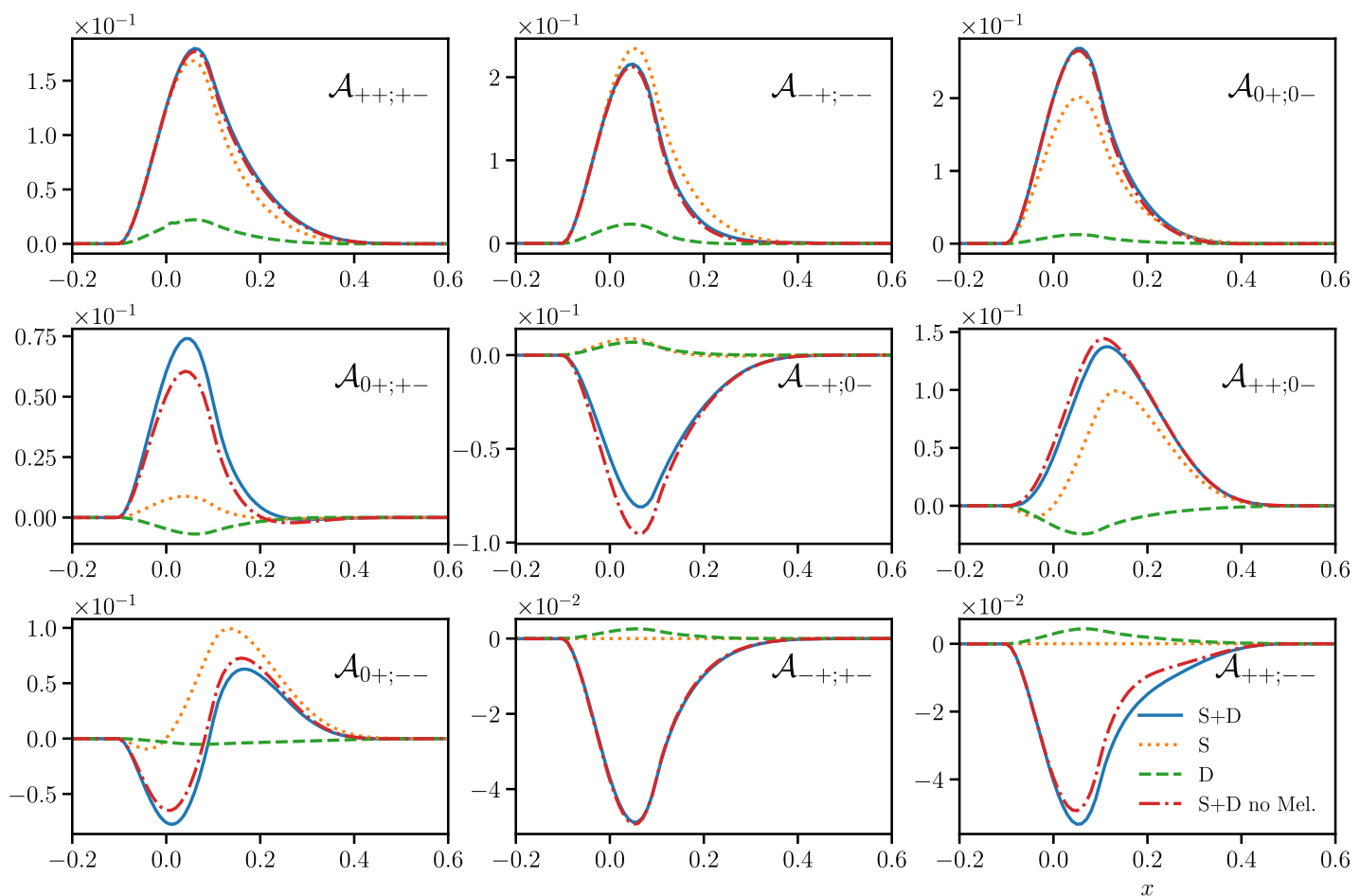

FIG. 3. Deuteron quark helicity amplitudes computed in the convolution formalism, at $\xi=0.1, t=-0.25 \mathrm{GeV}^{2}$. Full blue curve includes the full deuteron wave function, dotted orange (dashed green) only includes the deuteron radial $S$ - $(D$-)wave and the dasheddotted red curve omits the Melosh rotations in the light-front deuteron wave function.
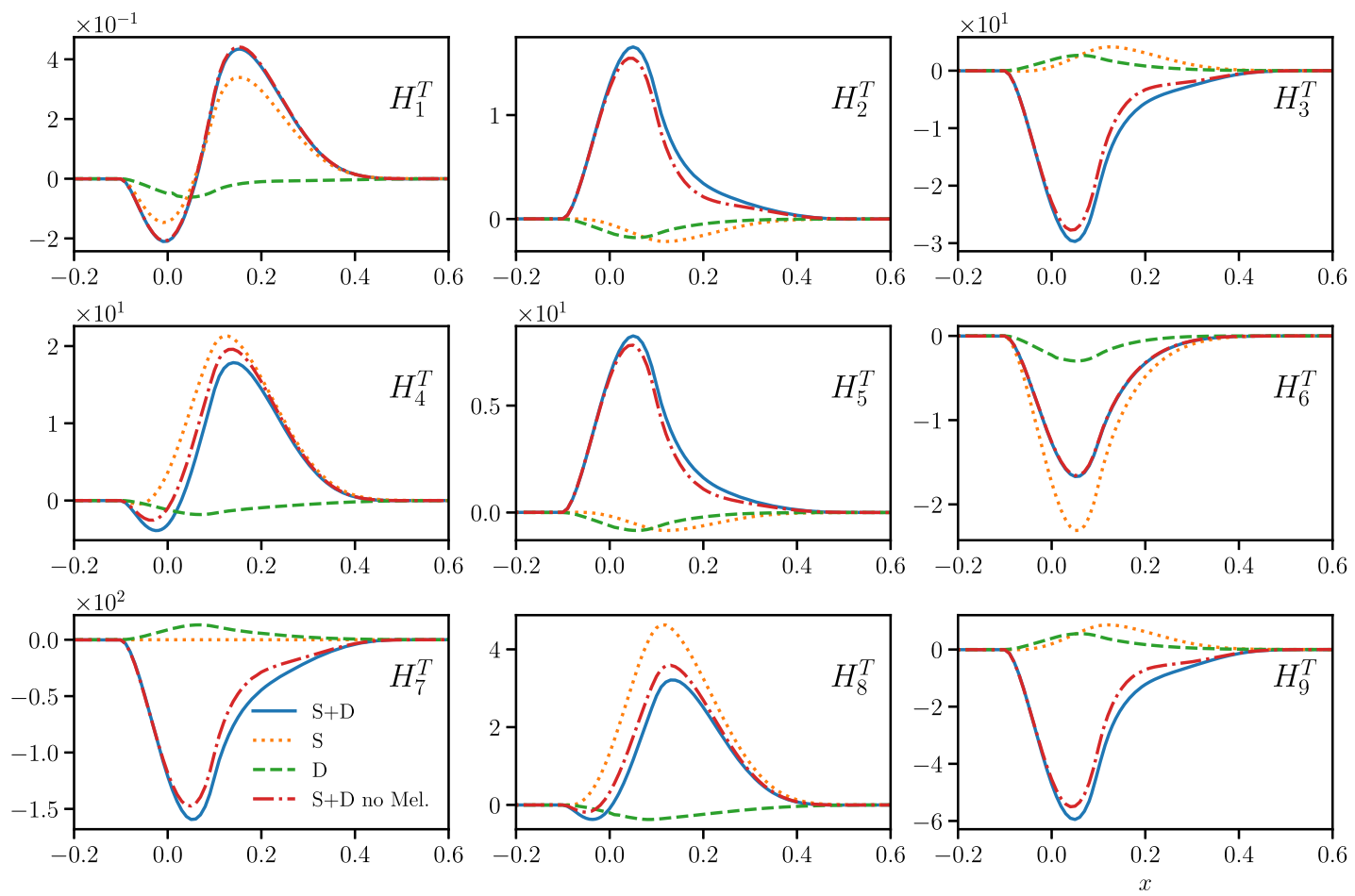

FIG. 4. Deuteron quark transversity GPDs computed in the convolution formalism, at $\xi=0.1, t=-0.25 \mathrm{GeV}^{2}$. Curves as in Fig. 3 . 

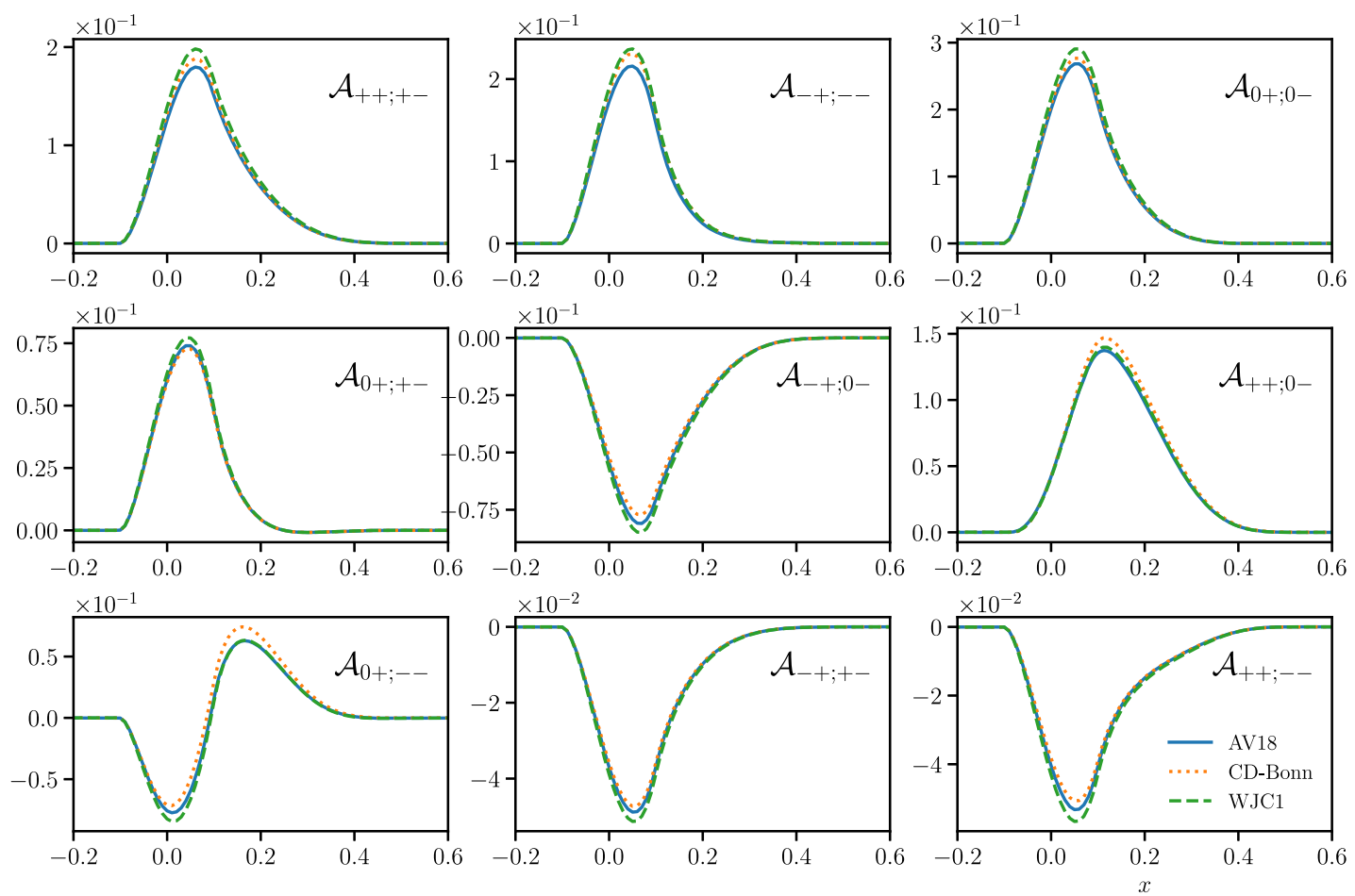

FIG. 5. Deuteron quark helicity amplitudes computed in the convolution formalism, at $\xi=0.1, t=-0.25 \mathrm{GeV}^{2}$ with different deuteron wave functions. Deuteron wave functions are CD-Bonn [42], WJC-1 [43] and AV18 [41].
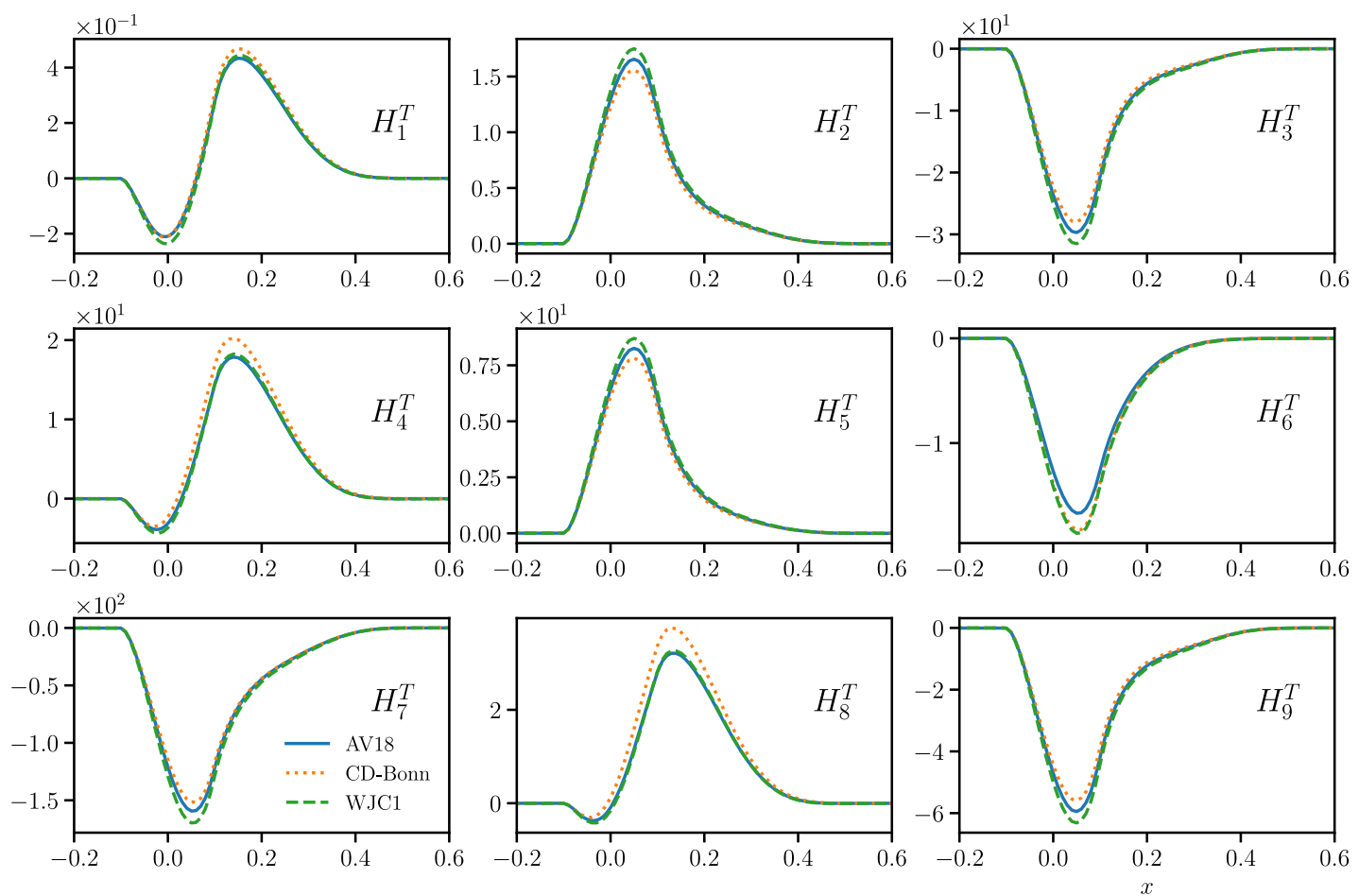

FIG. 6. Deuteron quark transversity GPDs computed in the convolution formalism, at $\xi=0.1, t=-0.25 \mathrm{GeV}^{2}$. Comparison between different wave functions. 

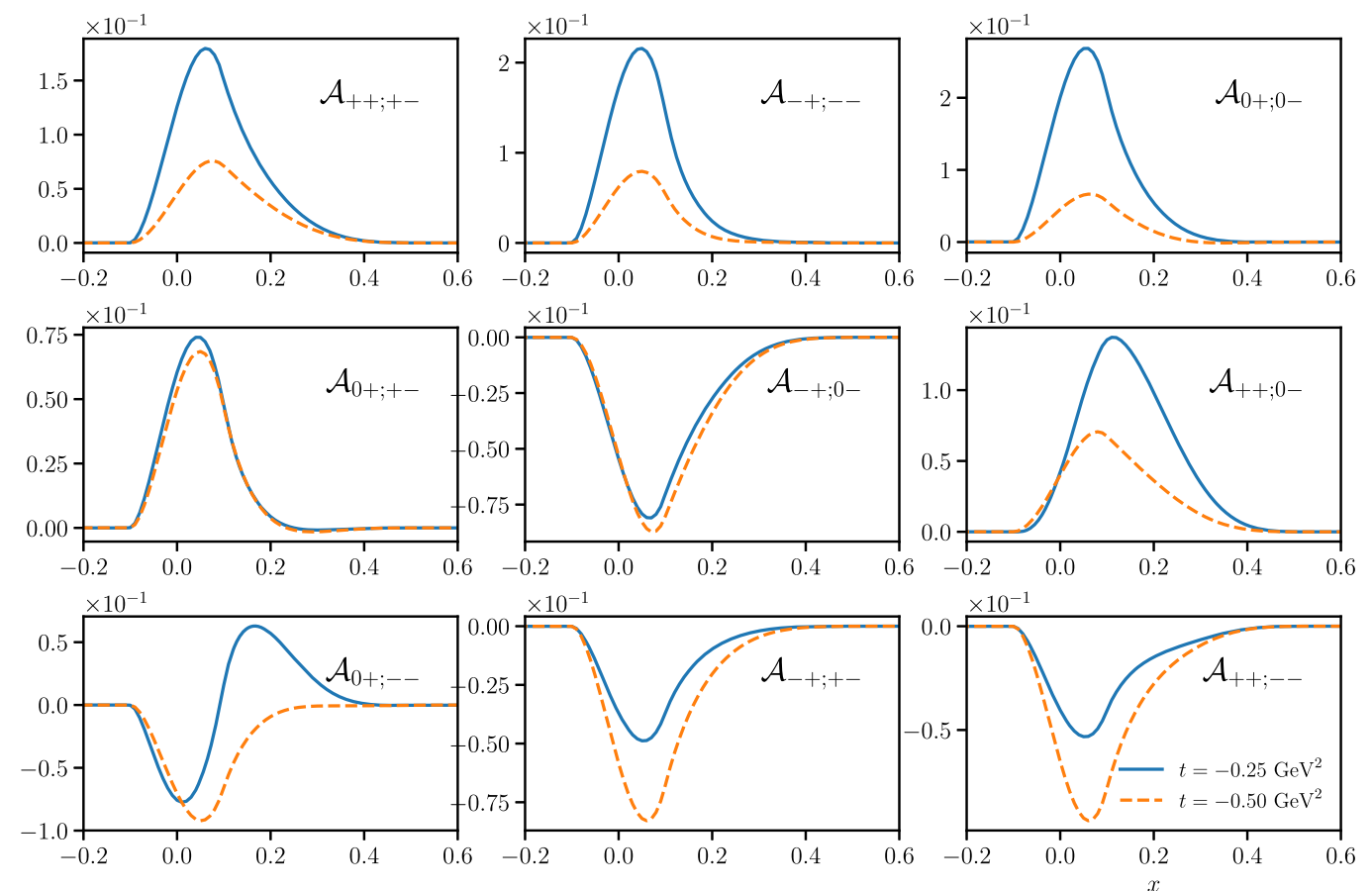

FIG. 7. Deuteron quark helicity amplitudes computed in the convolution formalism, at $\xi=0.1$ and two values of momentum transfer $t$.

panel) are identically zero for the $S$-wave as there is no orbital angular momentum available in the deuteron to compensate the change in helicities (two units for the deuteron, one unit for the quark).

We compared calculations with the three slightly different implementations of the chiral odd nucleon GPD models used in Ref. [38]. The results proved to be rather insensitive to these choices as changes in the final deuteron GPDs were in the order of a few percent maximum. Similarly, Figs. 5 and 6 show the use of three different deuteron wave functions in the calculation: the CD-Bonn [42] has a soft high-momentum tail, the WJC-1 [43] a hard one, and the
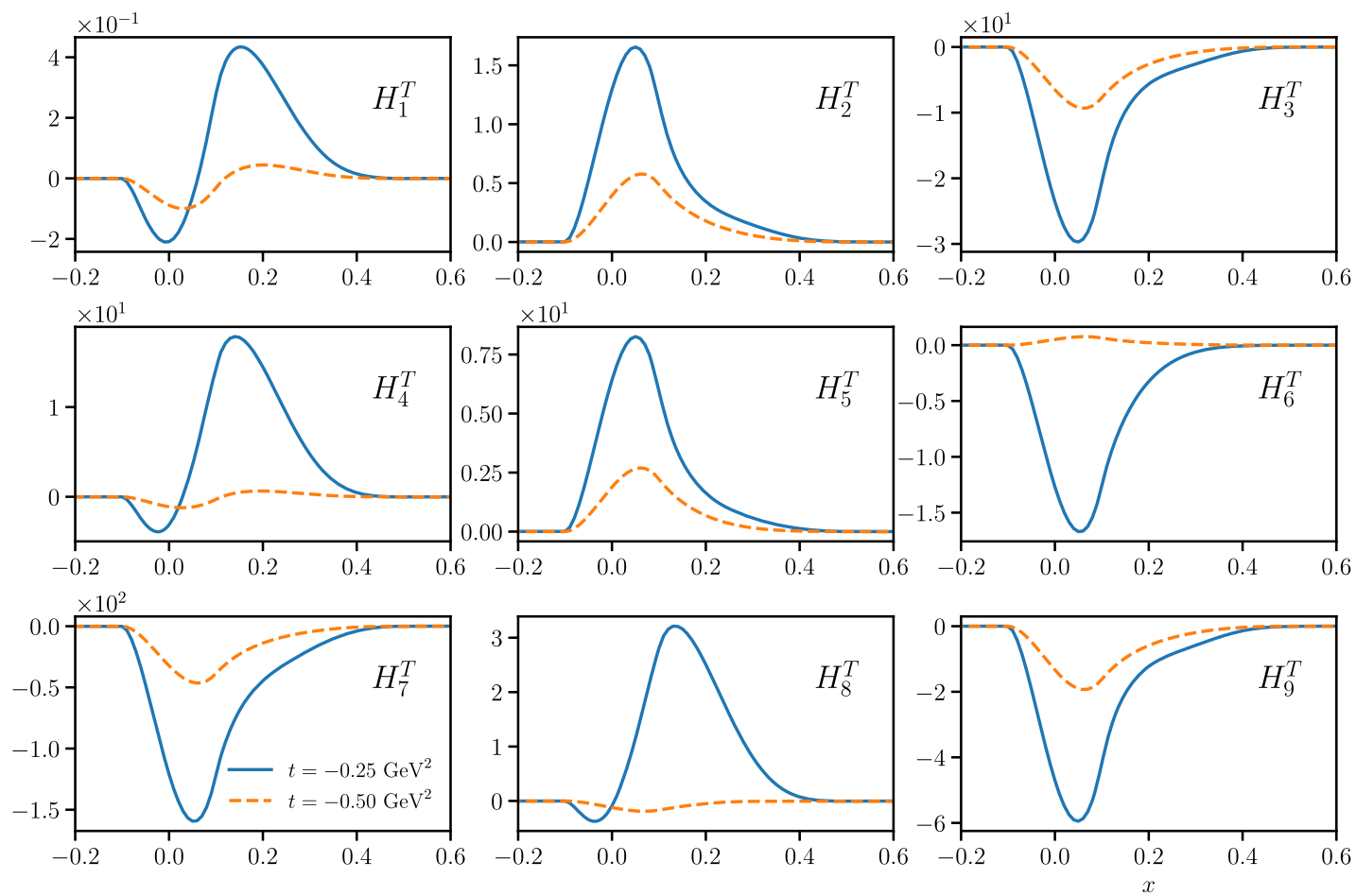

FIG. 8. Deuteron quark transversity GPDs computed in the convolution formalism, at $\xi=0.1$ and two values of momentum transfer $t$. 

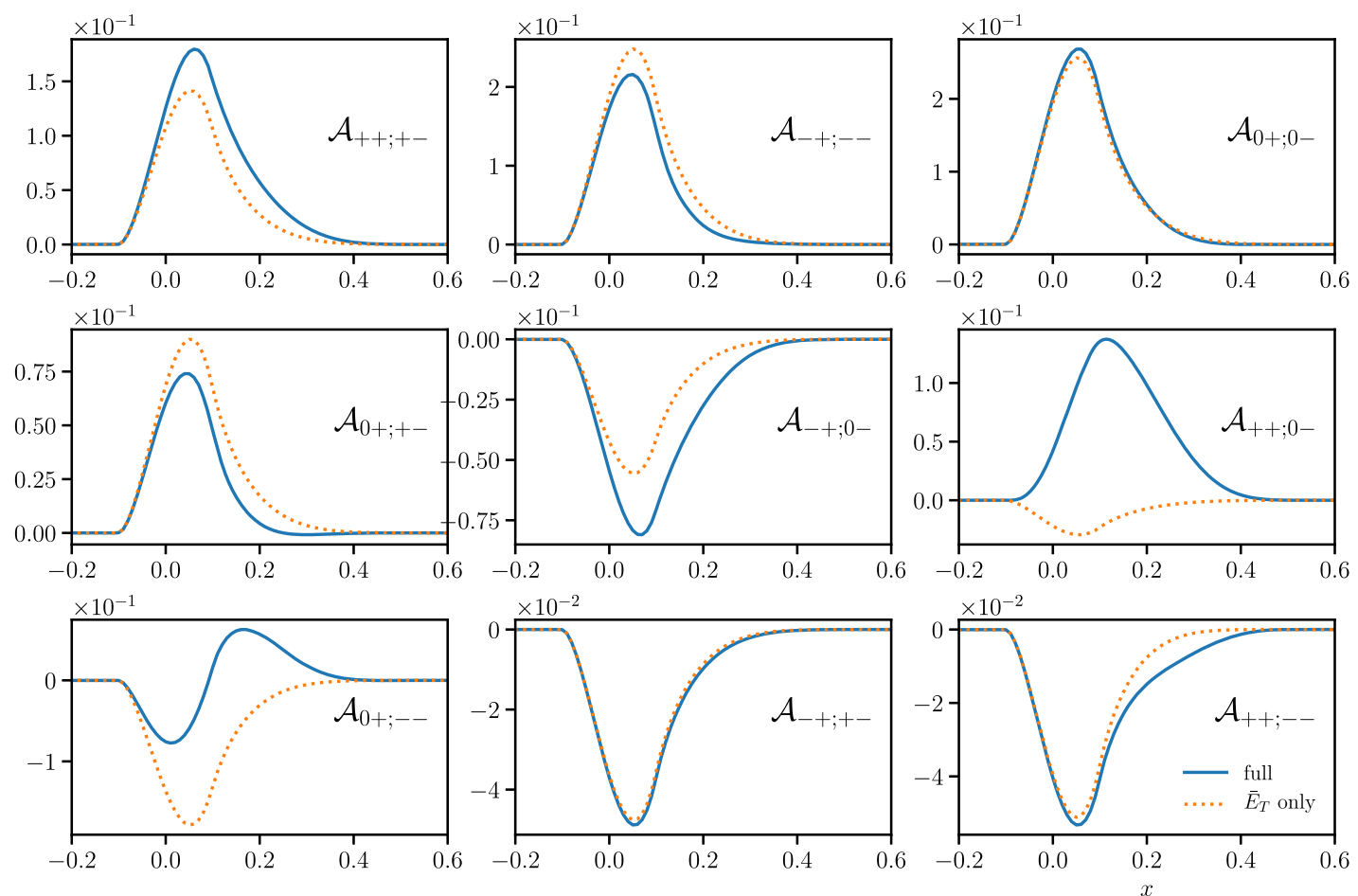

FIG. 9. Deuteron quark helicity amplitudes computed in the convolution formalism, at $\xi=0.1, t=-0.25 \mathrm{GeV}^{2}$. Dashed curve is a calculation only considering the $\bar{E}_{T}$ nucleon GPD.
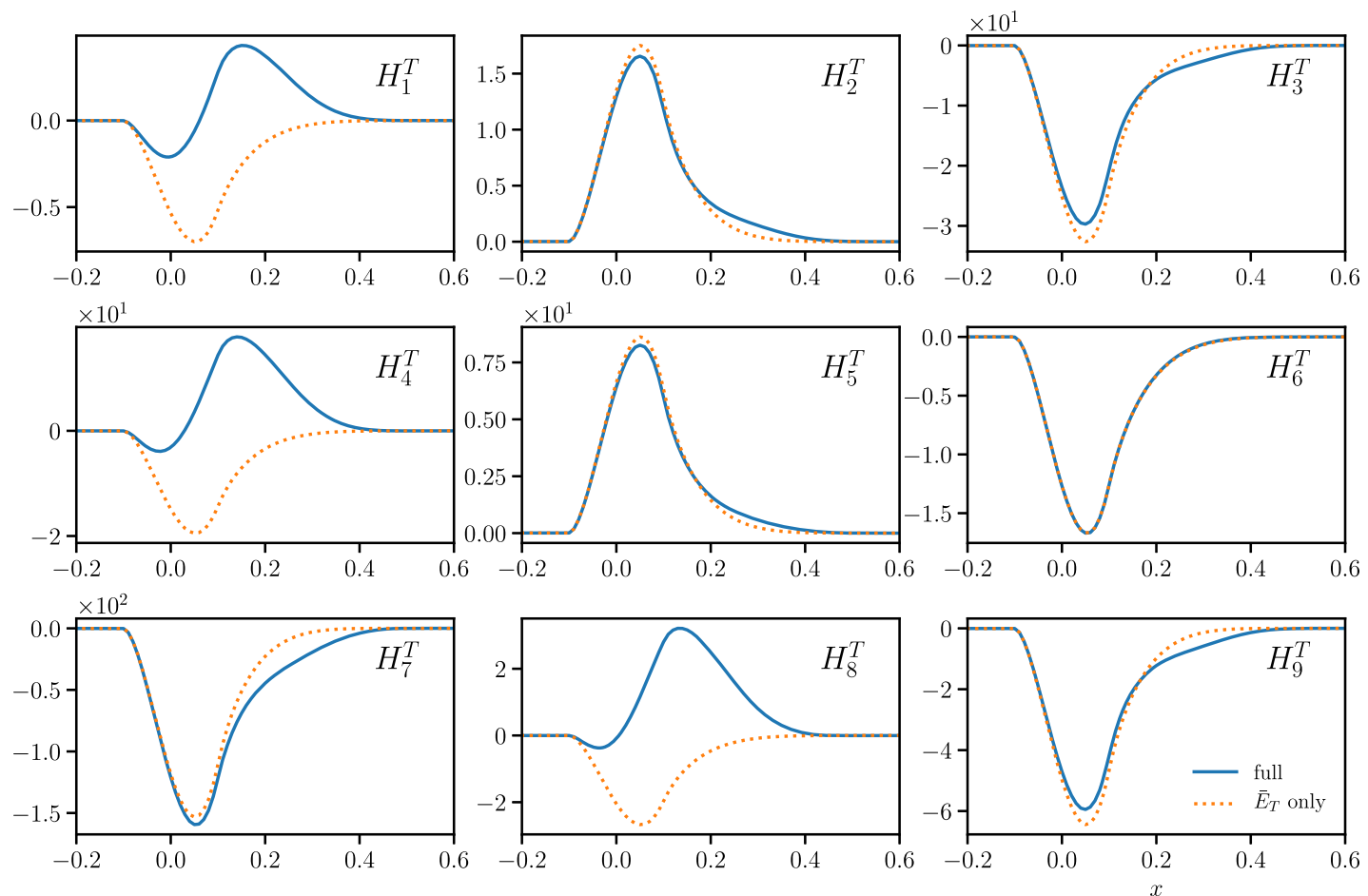

FIG. 10. Deuteron quark transversity GPDs computed in the convolution formalism, at $\xi=0.1, t=-0.25 \mathrm{GeV}^{2}$. Dashed curve is a calculation only considering the $\bar{E}_{T}$ nucleon GPD. 

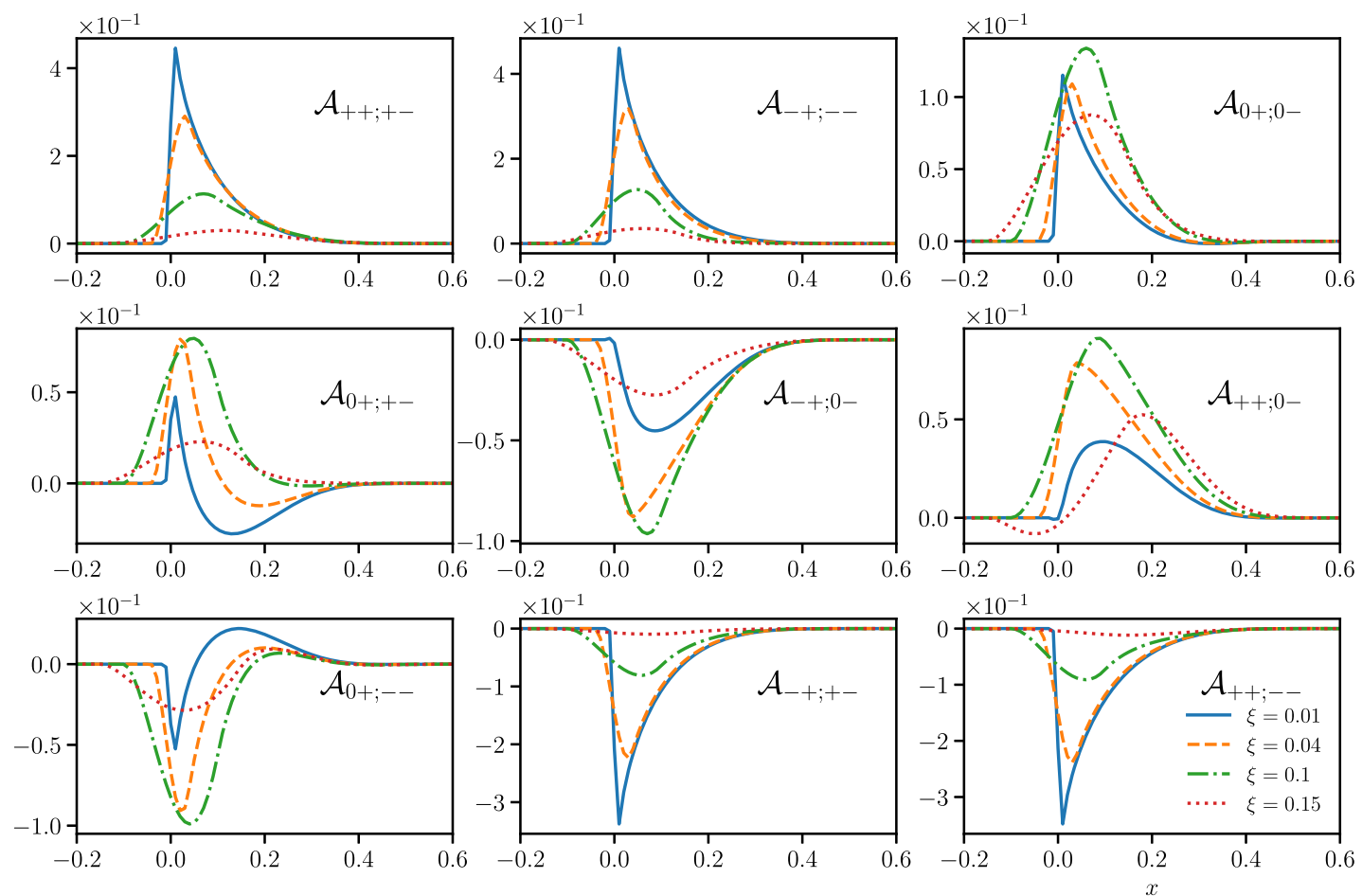

FIG. 11. Deuteron quark helicity amplitudes computed in the convolution formalism, at various $\xi$ for $t=-0.4 \mathrm{GeV}^{2}$.

AV18 [41] wave function is in between. Consequently, the differences between the different parametrizations included here are largest at high $\boldsymbol{p}^{\perp}$ or $\alpha_{1}$ close to its lower (0) and upper (2) bound. Both the amplitudes and GPDs are in general rather insensitive to the wave function details, even for the amplitudes that do not receive a pure $S$-wave contribution, and which are dominated by high relative $N N$ momenta in the convolution.
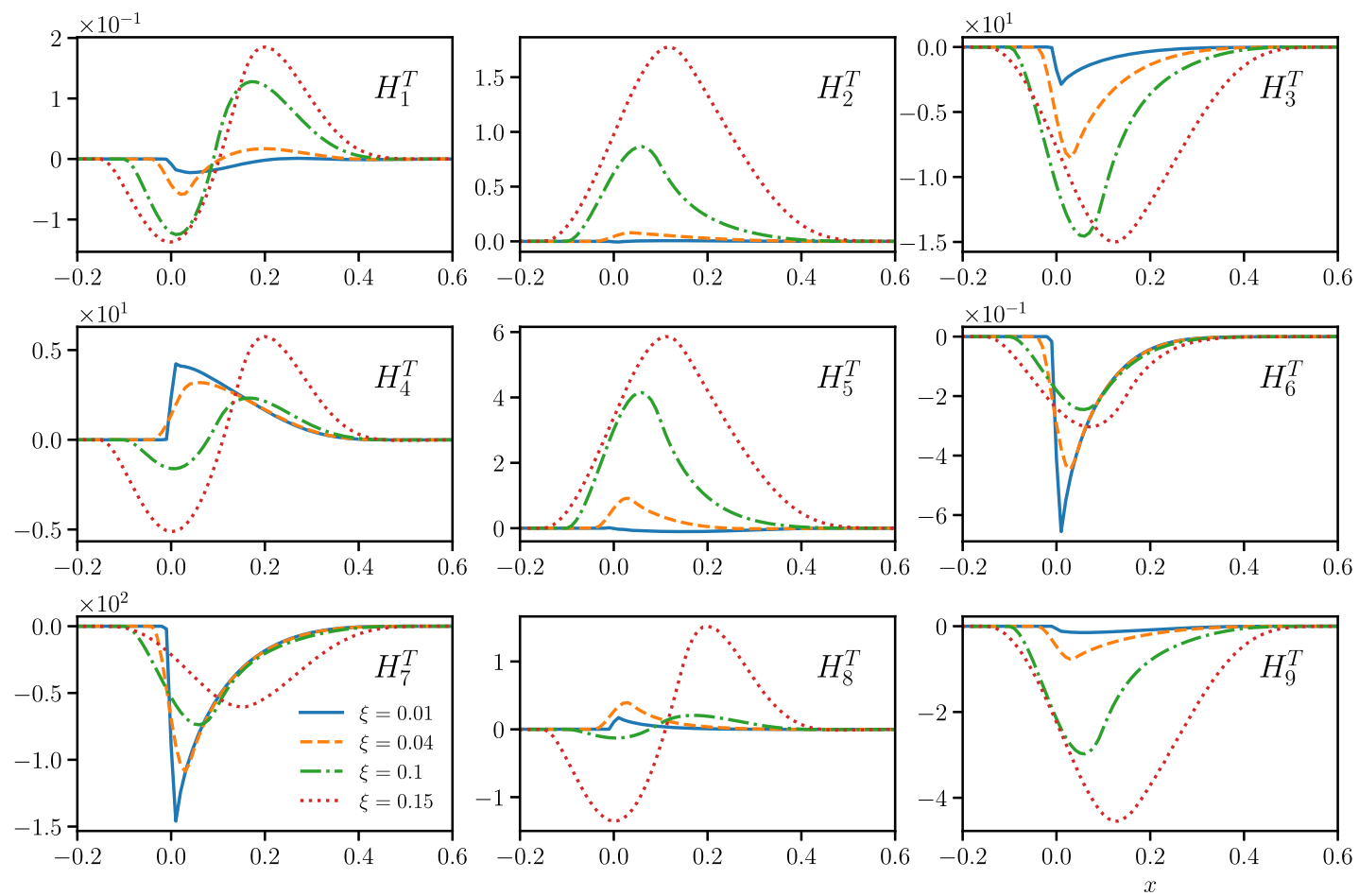

FIG. 12. Deuteron quark transversity GPDs computed in the convolution formalism, at various $\xi$ for $t=-0.4 \mathrm{GeV}^{2}$. 
Figures 7 and 8 show calculations at two values of momentum transfer. Helicity amplitudes without a deuteron helicity flip shrink in size with a higher momentum transfer. The amplitudes with a single helicity flip also become slightly smaller but the effect is not as large. Finally, the amplitudes with a double helicity flip grow in size. This reflects the role angular momentum plays in these amplitudes, being supplied by the momentum transfer. The GPDs are in general smaller at higher momentum transfer. $H_{6}^{T}$ has a flipped sign; this is caused by the fact that $H_{6}^{T}$ is proportional to the sum of helicity conserving and double helicity flip amplitudes (entering with a different sign) [Eqs. (C26) and (C28)].

Figures 9 and 10 show that most helicity amplitudes are dominated by the $\bar{E}_{T}=2 \tilde{H}_{T}+E_{T}$ nucleon chiral odd GPD from the GK parametrization. Only the $\mathcal{A}_{++; 0-}$ and $\mathcal{A}_{0+;--}$ receive large contributions from $H_{T}$. The dominance of $\bar{E}_{T}$ in most amplitudes is caused by its size on the one hand (which is larger than $H_{T}$ ) and the fact that both $u$ and $d$ quarks have same sign $\bar{E}_{T}$ GPDs, whereas they have opposite for $H_{T}$ and thus are small for the isosinglet contribution entering in the convolution formula.

Figures 11 and 12 show the $\xi$ dependence of the amplitudes and GPDs at a momentum transfer of $t=-0.4 \mathrm{GeV}^{2}$. The deuteron helicity amplitudes with zero or two units of deuteron helicity flip decrease significantly with larger $\xi$, while the ones with one unit of helicity flip are largest at intermediate values of $\xi$. For the GPDs, $H_{6}^{T}, H_{7}^{T}$ decrease significantly with larger $\xi$.

\section{SUM RULES IN THE DEUTERON CONVOLUTION PICTURE}

In this section we focus on the quark transversity GPD sum rules of Eq. (19). Because of Lorentz invariance, the GPDs obey polynomiality properties that in particular predict that these first moments should be independent of the value of skewness. As we use a lowest order Fock space expansion in our convolution model, and this explicitly breaks Lorentz invariance (no negative energy projections are included for instance), we investigate to which degree the $\xi$ independence is violated in our convolution formalism. Figure 13 depicts the results for the first moments of all the chiral odd quark GPDs at $t=-0.4 \mathrm{GeV}^{2}$ (which requires $|\xi|<0.17$ ). We see that several GPDs show a significant $\xi$ dependence, especially the GPDs $H_{3}^{T}, H_{4}^{T}, H_{5}^{T}$ and $H_{9}^{T}$. Two of these $\left(H_{3}^{T}, H_{5}^{T}\right)$ even should have zero first moments according to Eq. (19). This could be seen as a requirement to include higher order contributions in the convolution picture, i.e., beyond the handbag diagram or including higher Fock states.

To investigate this further, we look at the sum rules in a minimal convolution picture, detailed in Appendix D. This minimal convolution picture allows us to calculate the deuteron GPDs analytically. Looking at the final

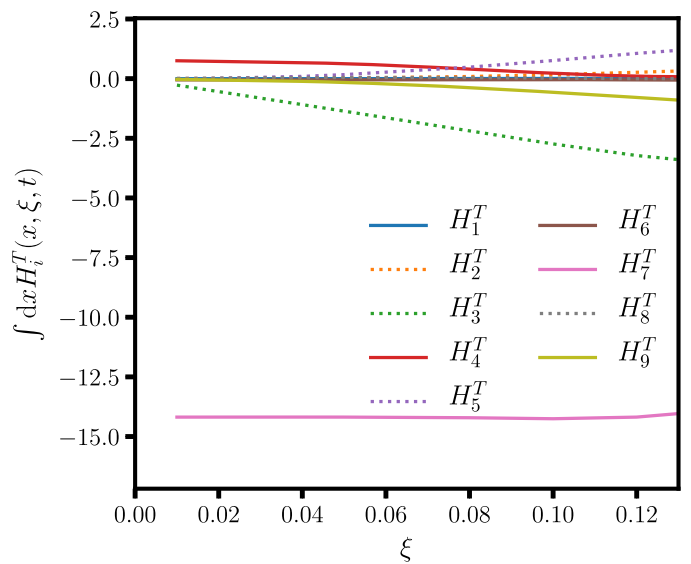

FIG. 13. First moments of the chiral odd quark GPDs at $t=-0.4 \mathrm{GeV}^{2}$ as a function of skewness $\xi$. Dashed curves are the GPDs that have zero sum rules. The moderate $\xi$ dependence is a consequence of Lorentz symmetry breaking due to lowest order approximation of the convolution picture.

expressions for the deuteron GPDs listed in Eqs. (D4), we see that only GPDs $H_{4}^{T}, H_{5}^{T}, H_{6}^{T}$ have a leading term $\mathcal{O}\left(\xi^{0}\right)$. Inspecting Eqs. (D4), almost all GPDs have dominating terms proportional to $D^{-2}$ (which is large for the deuteron kinematics considered here) that go as higher powers of $\xi$, especially the GPDs that also show the largest $\xi$ dependence in the full convolution model. It is worth noting that the fact that $H_{7}^{T}=0$ in this minimal convolution is due to the lack of a $D$-wave part in the deuteron wave function in this model and not a reflection of a sum rule.

The violation of the sum rules thus is an inherent feature of all convolution models based on a Fock space expansion, even the simplest ones. One should thus blame their formulation for Lorentz invariance breaking. The contribution of higher Fock states is beyond the current scope of our study. One possible approach for the deuteron that respects Lorentz invariance (and thus polynomiality of the GPDs) is the use of the covariant Bethe-Salpeter equation for the deuteron. Current deuteron GPD implementations of the Bethe-Salpeter approach are limited to a contact $N N$ interaction [44], while the approach presented here allows the use of realistic deuteron wave function parametrizations.

\section{CONCLUSION}

Our study completes the description of leading twist quark and gluon GPDs in the deuteron, in a convolution model based on the impulse approximation and using the lowest Fock space state for the deuteron in terms of nucleons. Although this picture is far from complete, it is a necessary starting point for the study of exclusive hard reactions in the QCD collinear factorization framework. It will enable us to confront this framework to near future experimental results. We showed that the GPDs were not 
very model-sensitive to the nucleon potential as far as the impulse approximation was used. However a richer structure as those involving a hidden color component [45] should lead to quite different GPDs, in particular in the gluonic sector.

The transversity sector is remarkably quite difficult to access in hard reactions [46], mostly because of the chiralodd character of the quark transversity distributions. As far as transversity quark GPDs are concerned, the fact that they do not contribute to the leading twist amplitude for the electroproduction of one meson $[47,48]$ lead to the study of higher twist $[37,49,50]$ or quark mass sensitive $[38,51]$ contributions, and to the study of other reactions with more particles in the final state [39,52-54]. The case for gluon transversity GPDs is rather different since they appear in the leading twist DVCS [55] and timelike Compton scattering [56] amplitudes.

We shall address the rich phenomenology of these reactions on the deuteron in future works, both for moderate energy range of JLab [57] and for the very high energy range aimed at the EIC [58] and the $\mathrm{LHeC} \mathrm{[59]} \mathrm{with}$ deuteron beams.

\section{ACKNOWLEDGMENTS}

We acknowledge useful discussions with Adam Freese, Cédric Lorcé, Claude Roiesnel, Lech Szymanowski, and Jakub Wagner. We thank Jakub Wagner for help with the numerical implementation of the chiral odd nucleon GPD parametrization.

\section{APPENDIX A: CONVENTIONS}

This Appendix summarizes the conventions and notations used throughout the text. We work with the following light-front conventions:

(i) Light-front components and Levi-Civita tensor

$$
x^{ \pm}=x^{0} \pm x^{3}, \quad \epsilon^{0123}=1 .
$$

(ii) We use the transverse $R / L$ indices defined as

$$
a^{R}=a^{x}+i a^{y}, \quad a^{L}=a^{x}-i a^{y},
$$

and for the action of light-front discrete symmetries we need the notation

$$
\tilde{a}^{\mu}=\left(a^{+}, a^{-},-a^{1}, a^{2}\right) .
$$

We have

$$
\tilde{a}^{R}=-a^{L}, \quad \tilde{a}^{L}=-a^{R} .
$$

The product of two four-vectors can be written as

$$
a^{\mu} b_{\mu}=\frac{1}{2}\left(a^{+} b^{-}+a^{-} b^{+}-a^{R} b^{L}-a^{L} b^{R}\right) .
$$

(iii) Single-particle state normalization of light-front helicity states

$$
\left\langle p^{\prime} \lambda^{\prime} \mid p \lambda\right\rangle=(2 \pi)^{3} 2 p^{+} \delta_{\lambda \lambda^{\prime}} \delta^{+\perp}\left(p-p^{\prime}\right) .
$$

(iv) Creation and annihilation operators are normalized through

$$
\left[a_{p}^{\lambda}, a_{p^{\prime}}^{\lambda^{\prime} \dagger}\right]_{ \pm}=(2 \pi)^{3} \delta_{\lambda \lambda^{\prime}} \delta^{+\perp}\left(p-p^{\prime}\right)
$$

(v) The last two equations imply

$$
|p \lambda\rangle=\sqrt{2 p^{+}} a_{p}^{\dagger \lambda}|0\rangle .
$$

(vi) The Dirac field in light-front quantization becomes

$$
\begin{aligned}
\psi(x)= & \sum_{\lambda= \pm \frac{1}{2}} \int \frac{d k^{+\perp}}{\sqrt{2 k^{+}}(2 \pi)^{3}} \\
& \times\left[a_{k}^{\lambda} u(k, \lambda) e^{-i k x}+b_{k}^{\dagger \lambda} v(k, \lambda) e^{i k x}\right],
\end{aligned}
$$

with $u(k, \lambda), v(k, \lambda)$ the standard light-front spinors [36].

(vii) The gluon field [with an implicit summation over a color index and $S U(3)$ generators implied]

$$
\begin{aligned}
A^{\mu}(x)= & \sum_{\lambda= \pm} \int \frac{d k^{+\perp}}{\sqrt{2 k^{+}}(2 \pi)^{3}} \\
& \times\left[a_{k}^{\lambda} \epsilon^{\mu}(k, \lambda) e^{-i k x}+a_{k}^{\dagger \lambda} \epsilon^{\mu *}(k, \lambda) e^{i k x}\right],
\end{aligned}
$$

where the polarization four-vectors are

$$
\begin{aligned}
& \epsilon^{\mu}(k,+)=\left[\begin{array}{cccc}
+ & - & 1 & 2 \\
0 & -\frac{\sqrt{2} k^{R}}{k^{+}} & -\frac{1}{\sqrt{2}} & -\frac{i}{\sqrt{2}}
\end{array}\right], \\
& \epsilon^{\mu}(k,-)=\left[\begin{array}{cccc}
+ & - & 1 & 2 \\
0 & \frac{\sqrt{2} k^{L}}{k^{+}} & \frac{1}{\sqrt{2}} & -\frac{i}{\sqrt{2}}
\end{array}\right] \text {. }
\end{aligned}
$$

Finally, the field strength and dual field strength are

$$
\begin{aligned}
& G^{\mu \nu}(x)=\partial^{\mu} A^{\nu}(x)-\partial^{\nu} A^{\mu}(x)-i g\left[A^{\mu}(x), A^{\nu}(x)\right] \\
& \tilde{G}^{\mu \nu}(x)=-\frac{1}{2} \epsilon^{\mu \nu \rho \sigma} G_{\rho \sigma} .
\end{aligned}
$$




\section{APPENDIX B: LIGHT-FRONT DISCRETE SYMMETRIES}

Light-front discrete symmetries were first considered in Ref. [60] and are discussed in several other instances of the literature with slightly different forms of the operators between them (see for instance in Refs. [61-63]). We follow the definitions used in Refs. $[62,63]$ as the combination of light-front parity and time reversal with the standard charge conjugation is consistent with the instant form $C P T$. To our knowledge, the action of these lightfront discrete symmetry operators on single-particle states and quark and gluon fields has not been summarized in detail, or in the case it has been written out [62], the intermediary formulas contain a number of errors and inconsistencies. We therefore include a summary here as a pedagogical Appendix.

\section{Light-front parity}

We can introduce the light-front parity symmetry transformation by its action on a coordinate four-vector,

$$
\Lambda\left(\mathcal{P}_{\perp}\right): x^{\mu} \mapsto \tilde{x}^{\mu}=\left(x^{+}, x^{-},-x^{1}, x^{2}\right) .
$$

As an operator there are a few possible choices to implement this transformation. These differ in an overall sign of the phase in the exponential, but do not yield differences when considering the action of $\mathcal{P}_{\perp}$ on correlator matrix elements. We choose

$$
\mathcal{P}_{\perp}=e^{-i \pi J_{1}} \mathcal{P}=e^{-i \frac{\pi}{2} J_{3}} e^{i \pi J_{2}} e^{i \frac{i \pi}{2} J_{3}} \mathcal{P}
$$

with $\mathcal{P}$ the standard instant form parity operator. We first consider the massive case. In the rest frame, acting with $\mathcal{P}_{\perp}$ on a massive single-particle state ${ }^{4}$ with spin $j$ yields using Eq. (B2)

$$
\mathcal{P}_{\perp}|j m\rangle=e^{-i \pi j} \eta|j m\rangle,
$$

where $\eta$ is the intrinsic parity of the particle. For light-front helicity states (defined with the standard light-front boosts) and using the commutation relations of the Lorentz group algebra, we obtain

$$
\mathcal{P}_{\perp}|p \lambda\rangle=e^{-i \pi j} \eta|\tilde{p}-\lambda\rangle
$$

Light-front parity thus flips the light-front helicity of the particle and transforms its momentum. For the creation and annihiliation operators we obtain

\footnotetext{
${ }^{4}$ The normalization of particle states and fields is given in Appendix A.
}

$$
\begin{aligned}
& \mathcal{P}_{\perp} a_{p}^{\dagger \lambda} \mathcal{P}_{\perp}^{\dagger}=\eta_{a} e^{-i \pi j} a_{\tilde{p}}^{\dagger-\lambda}, \\
& \mathcal{P}_{\perp} a_{p}^{\lambda} \mathcal{P}_{\perp}^{\dagger}=\eta_{a}^{*} e^{i \pi j} a_{\tilde{p}}^{-\lambda} .
\end{aligned}
$$

For the Dirac field, we have

$$
\begin{aligned}
\mathcal{P}_{\perp} \psi(x) \mathcal{P}_{\perp}^{\dagger}= & \sum_{\lambda= \pm \frac{1}{2}} \int \frac{d \tilde{k}^{+\perp}}{\sqrt{2 \tilde{k}^{+}}(2 \pi)^{3}}\left[\eta_{a}^{*} e^{i \pi j} a_{\tilde{k}}^{-\lambda} u(k, \lambda) e^{-i \tilde{k} \tilde{x}}\right. \\
& \left.+\eta_{b} e^{-i \pi j} b_{\tilde{k}}^{\dagger-\lambda} v(k, \lambda) e^{i \tilde{k} \tilde{x}}\right]
\end{aligned}
$$

The light-front spinors have

$$
\begin{aligned}
& \gamma^{1} \gamma_{5} u(\tilde{k},-\lambda)=u(k, \lambda), \\
& \gamma^{1} \gamma_{5} v(\tilde{k},-\lambda)=v(k, \lambda),
\end{aligned}
$$

and when requiring $\eta_{b}=-\eta_{a}^{*}$ as in the instant form case, we have

$$
\begin{aligned}
\mathcal{P}_{\perp} \psi(x) \mathcal{P}_{\perp}^{\dagger} & =\eta_{a}^{*} e^{i \pi j} \gamma^{1} \gamma^{5} \psi(\tilde{x}), \\
\mathcal{P}_{\perp} \psi^{\dagger}(x) \mathcal{P}_{\perp}^{\dagger} & =\eta_{a} e^{-i \pi j} \psi^{\dagger}(\tilde{x}) \gamma^{1} \gamma^{5} .
\end{aligned}
$$

When considering the transformation under $\mathcal{P}_{\perp}$ of the quark-quark correlators introduced in Eq. (9), the phases $e^{ \pm i \pi j}$ and intrinsic parities do not contribute as they cancel between the initial and final state and the two quark fields.

For massless states, the little group is characterized by a reference state with momentum along the $z$-axis $\bar{k}$ and two possible $J_{3}$ eigenvalues (if parity is a good symmetry). For this reference state one has

$$
\mathcal{P}_{\perp}|\bar{k} \lambda\rangle=\eta|\bar{k}-\lambda\rangle
$$

For a massless light-front helicity state with general momentum, this yields

$$
\mathcal{P}_{\perp}|p \lambda\rangle=\eta|\tilde{p}-\lambda\rangle
$$

so as in the massive case momentum transforms and lightfront helicity flips. Creation and annihilation operators transform as in Eq. (B5) but without the phase factor. For the gluon field this yields

$$
\begin{aligned}
\mathcal{P}_{\perp} A^{\mu}(x) \mathcal{P}_{\perp}^{\dagger}= & \sum_{\lambda= \pm} \int \frac{d \tilde{k}^{+\perp}}{\sqrt{2 \tilde{k}^{+}}(2 \pi)^{3}}\left[\eta_{a}^{*} a_{\tilde{k}}^{-\lambda} \epsilon^{\mu}(k, \lambda) e^{-i \tilde{k} \tilde{x}}\right. \\
& \left.+\eta_{a} a_{\tilde{k}}^{\dagger-\lambda} \epsilon^{\mu *}(k, \lambda) e^{i \tilde{k} \tilde{x}}\right] .
\end{aligned}
$$

The polarization four-vectors of Eq. (A11) have

$$
\epsilon^{\mu}(k, \pm)=\tilde{\epsilon}^{\mu}(\tilde{k}, \mp),
$$

and with $\eta_{a}$ real we have for the gluon field and field strength 


$$
\begin{aligned}
\mathcal{P}_{\perp} A^{\mu}(x) \mathcal{P}_{\perp}^{\dagger} & =\eta_{a} \tilde{A}^{\mu}(\tilde{x}), \\
\mathcal{P}_{\perp} G^{\mu \nu}(x) \mathcal{P}_{\perp}^{\dagger} & =\eta_{a} \bar{G}^{\mu \nu}(\tilde{x}),
\end{aligned}
$$

where $\bar{G}^{\mu \nu}(\tilde{x})=G^{\mu \nu}(\tilde{x})$ for an even number of indices 1 , and with a minus sign for an odd number. As in the quark case, the intrinsic parities and phases $e^{ \pm i \pi j}$ cancel in the light-front parity transformation of the gluon-gluon correlators of Eq. (10).

\section{Time reversal}

We can introduce the time reversal symmetry transformation by its action on a coordinate four-vector,

$$
\Lambda\left(\mathcal{T}_{\perp}\right): x^{\mu} \mapsto-\tilde{x}^{\mu}=\left(-x^{+},-x^{-}, x^{1},-x^{2}\right) .
$$

Because of the antiunitarity of $\mathcal{T}_{\perp}$ momenta transform as

$$
p^{\mu} \mapsto \tilde{p}^{\mu}=\left(p^{+}, p^{-},-p^{1}, p^{2}\right) .
$$

As with $\mathcal{P}_{\perp}$ there are several choices to write $\mathcal{T}_{\perp}$ on the operator level, with no difference at the level of transformation of correlator matrix elements. We take

$$
\mathcal{T}_{\perp}=e^{-i \pi J_{1}} \mathcal{T}=e^{-i \frac{\pi}{2} J_{3}} e^{i \pi J_{2}} e^{i \frac{\pi}{2} J_{3}} \mathcal{T}
$$

where $\mathcal{T}$ is the standard instant form time reversal operator. For massive particles, we have in the rest frame for a spin $j$ particle using Eq. (B16)

$$
\mathcal{T}_{\perp}|j m\rangle=e^{-i \pi m} \tilde{\eta}|j m\rangle,
$$

with $\tilde{\eta}$ a phase. For light-front helicity states we obtain

$$
\mathcal{T}_{\perp}|p \lambda\rangle=e^{-i \pi \lambda} \tilde{\eta}|\tilde{p} \lambda\rangle .
$$

Consequently light-front time reversal does not flip lightfront helicity, but momentum transforms. For the creation and annihilation operators we obtain

$$
\begin{aligned}
\mathcal{T}_{\perp} a_{p}^{\dagger \lambda} \mathcal{T}_{\perp}^{\dagger} & =\tilde{\eta}_{a} e^{-i \pi \lambda} a_{\tilde{p}}^{\dagger \lambda}, \\
\mathcal{T}_{\perp} a_{p}^{\lambda} \mathcal{T}_{\perp}^{\dagger} & =\tilde{\eta}_{a}^{*} e^{i \pi \lambda} a_{\tilde{p}}^{\lambda},
\end{aligned}
$$

and for the Dirac field one has

$$
\begin{aligned}
\mathcal{T}_{\perp} \psi(x) \mathcal{T}_{\perp}^{\dagger}= & \sum_{\lambda= \pm \frac{1}{2}} \int \frac{d \tilde{k}^{+\perp}}{\sqrt{2 \tilde{k}^{+}}(2 \pi)^{3}}\left[\tilde{\eta}_{a}^{*} e^{i \pi \lambda} a_{\tilde{k}}^{\lambda} u^{*}(k, \lambda) e^{-i \tilde{k}(-\tilde{x})}\right. \\
& \left.+\tilde{\eta}_{b} e^{-i \pi \lambda} b_{\tilde{k}}^{\dagger \lambda} v^{*}(k, \lambda) e^{i \tilde{k}(-\tilde{x})}\right] .
\end{aligned}
$$

The light-front spinors have

$$
\begin{aligned}
& -\gamma^{1} \gamma^{2} u(\tilde{k}, \lambda)=e^{i \pi \lambda} u^{*}(k, \lambda), \\
& -\gamma^{1} \gamma^{2} v(\tilde{k}, \lambda)=e^{-i \pi \lambda} v^{*}(k, \lambda),
\end{aligned}
$$

and when requiring $\tilde{\eta}_{b}=\tilde{\eta}_{a}^{*}$ as in the instant form case, we arrive at

$$
\begin{aligned}
\mathcal{T}_{\perp} \psi(x) \mathcal{T}_{\perp}^{\dagger} & =\tilde{\eta}_{a}^{*}\left(-\gamma^{1} \gamma^{2}\right) \psi(-\tilde{x}), \\
\mathcal{T}_{\perp} \psi^{\dagger}(x) \mathcal{T}_{\perp}^{\dagger} & =\tilde{\eta}_{a} \psi^{\dagger}(-\tilde{x})\left(-\gamma^{2} \gamma^{1}\right) .
\end{aligned}
$$

In the transformation under $\mathcal{T}_{\perp}$ of the quark-quark correlators of Eq. (9), all the phases cancel, but there remains an $(-1)^{\lambda^{\prime}-\lambda}$ factor originating from the transformation of the initial and final state [Eq. (B18)].

For the massless case, we have for the reference state

$$
\mathcal{T}_{\perp}|\bar{k} \lambda\rangle=\tilde{\eta}|\bar{k} \lambda\rangle
$$

and for the massless light-front helicity states with general momentum $p$

$$
\mathcal{T}_{\perp}|p \lambda\rangle=\tilde{\eta}|\tilde{p} \lambda\rangle
$$

Also in the massless case, light-front time reversal conserves light-front helicity and momentum is transformed. Creation and annihilation operators transform as in Eq. (B19) but without the phase factor. For the transformation of the gluon field, we arrive at

$$
\begin{aligned}
\mathcal{T}_{\perp} A^{\mu}(x) \mathcal{T}_{\perp}^{\dagger}= & \sum_{\lambda} \int \frac{d \tilde{k}^{+\perp}}{\sqrt{2 \tilde{k}^{+}}(2 \pi)^{3}}\left[\tilde{\eta}_{a}^{*} a_{\tilde{k}}^{\lambda} \epsilon^{\mu *}(k, \lambda) e^{-i \tilde{k}(-\tilde{x})}\right. \\
& \left.+\tilde{\eta}_{a} a_{\tilde{k}}^{\dagger \lambda} \epsilon^{\mu}(k, \lambda) e^{i \tilde{k}(-\tilde{x})}\right] .
\end{aligned}
$$

The polarization four-vectors of Eq. (7) have

$$
\epsilon^{\mu *}(k, \pm)=-\tilde{\epsilon}^{\mu}(\tilde{k}, \pm),
$$

and with $\tilde{\eta}_{a}$ real we have for the gluon field and field strength

$$
\begin{aligned}
\mathcal{T}_{\perp} A^{\mu}(x) \mathcal{T}_{\perp}^{\dagger} & =-\tilde{\eta}_{a} \tilde{A}^{\mu}(-\tilde{x}), \\
\mathcal{T}_{\perp} G^{\mu \nu}(x) \mathcal{T}_{\perp}^{\dagger} & =-\tilde{\eta}_{a} \bar{G}^{\mu \nu}(-\tilde{x}) .
\end{aligned}
$$

When considering the transformation of the gluon-gluon correlators of Eq. (10) with $\mathcal{T}_{\perp}$, the phases drop out but a factor $(-1)^{\lambda^{\prime}-\lambda}$ remains from the transformation of the initial/final state.

\section{APPENDIX C: EXPLICIT RELATIONS BETWEEN TRANSVERSITY GPDS AND HELICITY AMPLITUDES}

In the quark sector, the helicity amplitudes $\mathcal{A}_{\lambda^{\prime}+; \lambda-}^{q}$ can be written as a function of the nine transversity GPDs using Eq. (21), 


$$
\begin{aligned}
& \mathcal{A}_{++;+-}^{q}=-e^{i \phi} D\left[\frac{\xi}{1-\xi}\left(H_{3}^{q T}-H_{4}^{q T}\right)+H_{6}^{q T}+\frac{D^{2}}{2\left(1-\xi^{2}\right)} H_{7}^{q T}+\frac{1}{2(1-\xi)}\left(H_{8}^{q T}-H_{9}^{q T}\right)\right] \\
& \mathcal{A}_{0+; 0-}^{q}=e^{i \phi} D\left[-\frac{1}{2 \sqrt{2}}\left(H_{1}^{q T}+\xi H_{2}^{q T}\right)+\frac{2 \xi}{1-\xi^{2}} H_{3}^{q T}+\frac{2 D^{2}}{1-\xi^{2}} H_{4}^{q T}+\frac{1}{\sqrt{2}} H_{5}^{q T}+\frac{2 D^{2}-1-\xi^{2}}{1-\xi^{2}} H_{6}^{q T}\right. \\
& \left.+\frac{D^{4}-\xi^{2}}{\left(1-\xi^{2}\right)^{2}} H_{7}^{q T}-\frac{\xi}{1-\xi^{2}} H_{8}^{q T}-\frac{D^{2}}{1-\xi^{2}} H_{9}^{q T}\right] \\
& \mathcal{A}_{0+;+-}^{q}=\frac{e^{2 i \phi}}{\sqrt{2}}\left[\frac{(1-\xi)}{2 \sqrt{2}}\left(H_{1}^{q T}-H_{2}^{q T}\right)-\left(D^{2} \frac{1}{1-\xi}-2 \frac{\xi^{2}}{1-\xi^{2}}\right) H_{3}^{q T}+\left(D^{2} \frac{3 \xi-1}{1-\xi^{2}}-\frac{2 \xi^{2}}{1-\xi^{2}}\right) H_{4}^{q T}\right. \\
& +\frac{\xi}{\sqrt{2}}(1-\xi) H_{5}^{q T}-\frac{2 D^{2}}{1+\xi} H_{6}^{q T}-\frac{D^{2}}{(1+\xi)\left(1-\xi^{2}\right)}\left(D^{2}-\xi\right) H_{7}^{q T}+\left(-\frac{D^{2}}{2(1-\xi)}+\frac{\xi}{1-\xi^{2}}\right) H_{8}^{q T} \\
& \left.+\left(\frac{(3-\xi) D^{2}}{2\left(1-\xi^{2}\right)}-\frac{\xi}{1-\xi^{2}}\right) H_{9}^{q T}\right] \\
& \mathcal{A}_{++; 0-}^{q}=\frac{D^{2}}{\sqrt{2}(1-\xi)}\left[-H_{3}^{q T}+H_{4}^{q T}+2 H_{6}^{q T}+\frac{D^{2}+\xi}{1-\xi^{2}} H_{7}^{q T}+\frac{1}{2}\left(H_{8}^{q T}-H_{9}^{q T}\right)\right] \\
& \mathcal{A}_{-+;+-}^{q}=e^{3 i \phi} \frac{D}{1-\xi^{2}}\left[2 \xi\left(H_{3}^{q T}-\xi H_{4}^{q T}\right)+\frac{D^{2}}{2} H_{7}^{q T}+\left(\xi H_{8}^{q T}-H_{9}^{q T}\right)\right] \\
& \mathcal{A}_{++;--}^{q}=e^{-i \phi} \frac{D^{3}}{2\left(1-\xi^{2}\right)} H_{7}^{q T} .
\end{aligned}
$$

The other three helicity amplitudes $\mathcal{A}_{\lambda^{\prime}+; \lambda-}^{q}$ can also be obtained by applying Eq. (16) to the ones obtained above,

$$
\begin{aligned}
\mathcal{A}_{-+;--}^{q}= & -e^{i \phi} D\left[\frac{\xi}{1+\xi}\left(H_{3}^{q T}+H_{4}^{q T}\right)+H_{6}^{q T}+\frac{D^{2}}{2\left(1-\xi^{2}\right)} H_{7}^{q T}-\frac{1}{2(1+\xi)}\left(H_{8}^{q T}+H_{9}^{q T}\right)\right] \\
\mathcal{A}_{-+; 0-}^{q}= & \frac{e^{2 i \phi}}{\sqrt{2}}\left[\frac{(1+\xi)}{2 \sqrt{2}}\left(H_{1}^{q T}+H_{2}^{q T}\right)+\left(D^{2} \frac{1}{1+\xi}-2 \frac{\xi^{2}}{1-\xi^{2}}\right) H_{3}^{q T}-\left(D^{2} \frac{3 \xi+1}{1-\xi^{2}}+\frac{2 \xi^{2}}{1-\xi^{2}}\right) H_{4}^{q T}\right. \\
& -\frac{\xi}{\sqrt{2}}(1+\xi) H_{5}^{q T}-\frac{2 D^{2}}{1-\xi} H_{6}^{q T}-\frac{D^{2}}{(1-\xi)\left(1-\xi^{2}\right)}\left(D^{2}+\xi\right) H_{7}^{q T} \\
& \left.+\left(\frac{D^{2}}{2(1+\xi)}+\frac{\xi}{1-\xi^{2}}\right) H_{8}^{q T}+\left(\frac{(3+\xi) D^{2}}{2\left(1-\xi^{2}\right)}+\frac{\xi}{1-\xi^{2}}\right) H_{9}^{q T}\right] \\
\mathcal{A}_{0+;--}^{q}= & \frac{D^{2}}{\sqrt{2}(1+\xi)}\left[H_{3}^{q T}+H_{4}^{q T}+2 H_{6}^{q T}+\frac{D^{2}-\xi}{1-\xi^{2}} H_{7}^{q T}-\frac{1}{2}\left(H_{8}^{q T}+H_{9}^{q T}\right)\right] .
\end{aligned}
$$

The determinant of the matrix relating the helicity amplitudes and the GPDs in the above equations yields

$$
\operatorname{Det}_{q}=-\frac{1}{2^{9 / 2}} e^{9 i \phi} D^{11},
$$

which shows that all tensor structures appearing in Eq. (9) are linearly independent away from the forward limit.

For the gluon helicity amplitudes we obtain largely similar expressions as the tensors that are used in the decomposition are very similar. The main differences are (i) the right-hand side of all equations is multiplied with an extra $e^{i \phi} 2 D$ factor compared to the quark helicity amplitudes and (ii) there are differences for the factors multiplying the $H_{5}^{q T}$ and $H_{6}^{q T}$ GPDs as a different tensor structure was used, 


$$
\begin{aligned}
& \mathcal{A}_{++;+-}^{g}=-e^{2 i \phi} D^{2}\left[\frac{2 \xi}{1-\xi}\left(H_{3}^{g T}-H_{4}^{g T}\right)+\frac{D^{2}}{1-\xi^{2}} H_{7}^{g T}+\frac{1}{1-\xi}\left(H_{8}^{g T}-H_{9}^{g T}\right)\right] \\
& \mathcal{A}_{0+; 0-}^{g}=e^{2 i \phi} 2 D^{2}\left[-\frac{1}{2 \sqrt{2}}\left(H_{1}^{g T}+\xi H_{2}^{g T}\right)+\frac{2 \xi}{1-\xi^{2}} H_{3}^{g T}+\frac{2 D^{2}}{1-\xi^{2}} H_{4}^{g T}+\frac{1}{4}\left(H_{5}^{g T}+H_{6}^{g T}\right)\right. \\
& \left.+\frac{D^{4}-\xi^{2}}{(1-\xi)^{2}} H_{7}^{g T}-\frac{\xi}{1-\xi^{2}} H_{8}^{g T}-\frac{D^{2}}{1-\xi^{2}} H_{9}^{g T}\right] \\
& \mathcal{A}_{0+;+-}^{g}=e^{3 i \phi} \sqrt{2} D\left[\frac{1-\xi}{2 \sqrt{2}}\left(H_{1}^{g T}-H_{2}^{g T}\right)-\left(D^{2} \frac{1}{1-\xi}-2 \frac{\xi^{2}}{1-\xi^{2}}\right) H_{3}^{g T}+\left(D^{2} \frac{3 \xi-1}{1-\xi^{2}}-\frac{2 \xi^{2}}{1-\xi^{2}}\right) H_{4}^{g T}-\frac{1}{2}\left(H_{5}^{g T}-\xi_{6}^{g T}\right)\right. \\
& \left.-\frac{D^{2}}{(1+\xi)\left(1-\xi^{2}\right)}\left(D^{2}-\xi\right) H_{7}^{g T}+\left(-\frac{D^{2}}{2(1-\xi)}+\frac{\xi}{1-\xi^{2}}\right) H_{8}^{g T}+\left(\frac{(3-\xi) D^{2}}{2\left(1-\xi^{2}\right)}-\frac{\xi}{1-\xi^{2}}\right) H_{9}^{g T}\right] \\
& \mathcal{A}_{++; 0-}^{g}=e^{i \phi} \frac{\sqrt{2} D^{3}}{1-\xi}\left[-H_{3}^{g T}+H_{4}^{g T}+\frac{D^{2}+\xi}{1-\xi^{2}} H_{7}^{g T}+\frac{1}{2}\left(H_{8}^{g T}-H_{9}^{g T}\right)\right] \\
& \mathcal{A}_{-+;+-}^{g}=\frac{4 \xi D^{2}}{1-\xi^{2}}\left(H_{3}^{g T}-\xi H_{4}^{g T}\right)+e^{4 i \phi}\left(H_{5}^{g T}-\xi^{2} H_{6}^{g T}\right)+e^{4 i \phi} \frac{D^{2}}{1-\xi^{2}}\left[D^{2} H_{7}^{g T}+2\left(\xi H_{8}^{g T}-H_{9}^{g T}\right)\right] \\
& \mathcal{A}_{++;--}^{g}=\frac{D^{4}}{1-\xi^{2}} H_{7}^{g T}
\end{aligned}
$$

The other three helicity amplitudes $\mathcal{A}_{\lambda^{\prime}+; \lambda-}^{g}$ can be obtained by using Eq. (16),

$$
\begin{aligned}
\mathcal{A}_{-+;--}^{g}= & -e^{2 i \phi} D^{2}\left[2 \frac{\xi}{1+\xi}\left(H_{3}^{g T}+H_{4}^{g T}\right)+\frac{D^{2}}{1-\xi^{2}} H_{7}^{g T}-\frac{1}{1+\xi}\left(H_{8}^{g T}+H_{9}^{g T}\right)\right] \\
\mathcal{A}_{-+; 0-}^{g}= & e^{3 i \phi \sqrt{2} D}\left[\frac{1+\xi}{2 \sqrt{2}}\left(H_{1}^{g T}+H_{2}^{g T}\right)+\left(D^{2} \frac{1}{1+\xi}-2 \frac{\xi^{2}}{1-\xi^{2}}\right) H_{3}^{g T}-\left(D^{2} \frac{3 \xi+1}{1-\xi^{2}}+\frac{2 \xi^{2}}{1-\xi^{2}}\right) H_{4}^{g T}-\frac{1}{2}\left(H_{5}^{g T}+\xi H_{6}^{g T}\right)\right. \\
& \left.-\frac{D^{2}}{(1-\xi)\left(1-\xi^{2}\right)}\left(D^{2}+\xi\right) H_{7}^{g T}+\left(\frac{D^{2}}{2(1+\xi)}+\frac{\xi}{1-\xi^{2}}\right) H_{8}^{g T}+\left(\frac{(3+\xi) D^{2}}{2\left(1-\xi^{2}\right)}+\frac{\xi}{1-\xi^{2}}\right) H_{9}^{g T}\right] \quad(\mathrm{C} 18) \\
\mathcal{A}_{0+;--}^{g}= & e^{i \phi} \frac{\sqrt{2} D^{3}}{1+\xi}\left[H_{3}^{g T}+H_{4}^{g T}+\frac{D^{2}-\xi}{1-\xi^{2}} H_{7}^{g T}-\frac{1}{2}\left(H_{8}^{g T}+H_{9}^{g T}\right)\right] .
\end{aligned}
$$

The determinant of the above set of equations yields

$$
\operatorname{Det}_{g}=-2 e^{18 i \phi} D^{18}
$$

which is again nonzero away from the forward limit.

For completeness, we also list the inverse relations for both quarks and gluons as these are used to obtain the deuteron GPDs from the helicity amplitudes calculated in the convolution formalism.

For the quark GPDs we have

$$
\begin{aligned}
H_{1}^{q T}= & {\left[\frac{2 \sqrt{2} e^{-i \phi} \xi}{D\left(1-\xi^{2}\right)}\left(\mathcal{A}_{++;+-}^{q}-\mathcal{A}_{-+;--}^{q}\right)+2 e^{-2 i \phi}\left(\frac{1}{1-\xi} \mathcal{A}_{0+;+-}^{q}+\frac{1}{1+\xi} \mathcal{A}_{-+; 0-}^{q}\right)+\frac{2}{1+\xi} \mathcal{A}_{++; 0-}^{q}\right.} \\
& \left.+\frac{2}{1-\xi} \mathcal{A}_{0+;--}^{q}+\frac{2 \sqrt{2} D}{\left(1-\xi^{2}\right)}\left(e^{-3 i \phi} \mathcal{A}_{-+;+-}^{q}-e^{i \phi} \mathcal{A}_{++;--}^{q}\right)\right]
\end{aligned}
$$




$$
\begin{aligned}
H_{2}^{q T}= & {\left[\frac{\sqrt{2} e^{-i \phi}}{D(1+\xi)^{2}}\left(\frac{2 D^{2}}{1-\xi}-\xi\right) \mathcal{A}_{++;+-}^{q}-\frac{\sqrt{2} e^{-i \phi}}{D(1-\xi)^{2}}\left(\frac{2 D^{2}}{1+\xi}+\xi\right) \mathcal{A}_{-+;--}^{q}+\frac{2 \sqrt{2} e^{-i \phi} \xi}{\left(1-\xi^{2}\right) D} \mathcal{A}_{0+; 0-}^{q}\right.} \\
& -\frac{2 e^{-2 i \phi}}{1-\xi^{2}}\left(\mathcal{A}_{0+;+-}^{q}-\mathcal{A}_{-+; 0-}^{q}\right)+2\left(\frac{1}{(1+\xi)^{2}}+\frac{2 \xi^{2}}{D^{2}\left(1-\xi^{2}\right)(1+\xi)}\right) \mathcal{A}_{++; 0-}^{q} \\
& -2\left(\frac{1}{(1-\xi)^{2}}+\frac{2 \xi^{2}}{D^{2}\left(1-\xi^{2}\right)(1-\xi)}\right) \mathcal{A}_{0+;--}^{q}+\sqrt{2} \frac{e^{-3 i \phi} \xi}{D\left(1-\xi^{2}\right)} \mathcal{A}_{-+;+-}^{q} \\
& \left.-\frac{\sqrt{2} e^{i \phi} \xi}{D^{3}\left(1-\xi^{2}\right)^{2}}\left(4 \xi^{2}+D^{2}\left(3+\xi^{2}\right)\right) \mathcal{A}_{++;--}^{q}\right]
\end{aligned}
$$$$
H_{3}^{q T}=\left[-\frac{e^{-i \phi}}{2 D}\left(\frac{1-\xi}{1+\xi} \mathcal{A}_{++;+-}^{q}-\frac{1+\xi}{1-\xi} \mathcal{A}_{-+;--}^{q}\right)-\frac{1}{\sqrt{2} D^{2}}\left(\frac{1-\xi}{1+\xi} \mathcal{A}_{++; 0-}^{q}-\frac{1+\xi}{1-\xi} \mathcal{A}_{0+;--}^{q}\right)+\frac{2 e^{i \phi} \xi}{D^{3}\left(1-\xi^{2}\right)} \mathcal{A}_{++;--}^{q}\right],
$$$$
H_{4}^{q T}=\left[\frac{e^{-i \phi}}{D}\left(\frac{1}{1+\xi} \mathcal{A}_{++;+-}^{q}+\frac{1}{1-\xi} \mathcal{A}_{-+;--}^{q}\right)+\frac{1}{\sqrt{2} D^{2}}\left(\frac{1-\xi}{1+\xi} \mathcal{A}_{++; 0-}^{q}+\frac{1+\xi}{1-\xi} \mathcal{A}_{0+;--}^{q}\right)+\frac{e^{-3 i \phi}}{2 D} \mathcal{A}_{-+;+-}^{q}\right.
$$$$
-\frac{e^{i \phi}}{2 D^{3}}\left(D^{2}-\frac{4 \xi^{2}}{1-\xi^{2}}\right) \mathcal{A}_{++;--}^{q} \text {, }
$$$$
H_{5}^{q T}=\frac{1}{\sqrt{2}}\left[-\frac{e^{-i \phi}}{D}\left(\frac{1}{(1+\xi)^{2}}\left(\frac{1}{2}+\frac{D^{2}}{1-\xi}\right) \mathcal{A}_{++;+-}^{q}+\frac{1}{(1-\xi)^{2}}\left(\frac{1}{2}+\frac{D^{2}}{1+\xi}\right) \mathcal{A}_{-+;--}^{q}\right)+\frac{e^{-i \phi}}{\left(1-\xi^{2}\right) D} \mathcal{A}_{0+; 0-}^{q}\right.
$$$$
+\frac{e^{-2 i \phi}}{\sqrt{2}\left(1-\xi^{2}\right)}\left(\mathcal{A}_{0+;+-}^{q}+\mathcal{A}_{-+; 0-}^{q}\right)-\frac{1}{\sqrt{2}(1+\xi)^{2}}\left(1-\frac{2 \xi}{D^{2}(1-\xi)}\right) \mathcal{A}_{++; 0-}^{q}
$$$$
-\frac{1}{\sqrt{2}(1-\xi)^{2}}\left(1+\frac{2 \xi}{D^{2}(1+\xi)}\right) \mathcal{A}_{0+;--}^{q}-\frac{e^{-3 i \phi}}{2 D\left(1-\xi^{2}\right)} \mathcal{A}_{-+;+-}^{q}-\frac{e^{i \phi}\left(D^{2}\left(3 \xi^{2}+1\right)+4 \xi^{2}\right)}{2 D^{3}\left(1-\xi^{2}\right)^{2}} \mathcal{A}_{++;--}^{q},
$$$$
H_{6}^{q T}=-\frac{1}{2 D}\left[e^{-i \phi}\left(\mathcal{A}_{++;+-}^{q}+\mathcal{A}_{-+;--}^{q}\right)+e^{-3 i \phi} \mathcal{A}_{-+;+-}^{q}+e^{i \phi} \mathcal{A}_{++;--}^{q}\right],
$$$$
H_{7}^{q T}=\frac{2 e^{i \phi}\left(1-\xi^{2}\right)}{D^{3}} \mathcal{A}_{++;--}^{q},
$$$$
H_{8}^{q T}=\frac{1}{D}\left[-e^{-i \phi}\left(\frac{1-\xi}{1+\xi} \mathcal{A}_{++;+-}^{q}-\frac{1+\xi}{1-\xi} \mathcal{A}_{-+;--}^{q}\right)+\frac{\sqrt{2} \xi}{D}\left(\frac{1-\xi}{1+\xi} \mathcal{A}_{++; 0-}^{q}+\frac{1+\xi}{1-\xi} \mathcal{A}_{-+; 0-}^{q}\right)+\frac{4 e^{i \phi} \xi^{3}}{D^{2}\left(1-\xi^{2}\right)} \mathcal{A}_{++;--}^{q}\right],
$$$$
H_{9}^{q T}=-\frac{1}{D}\left[2 e^{-i \phi} \xi\left(\frac{1}{1+\xi} \mathcal{A}_{++;+-}^{q}-\frac{1}{1-\xi} \mathcal{A}_{-+;--}^{q}\right)+\frac{\sqrt{2} \xi}{D}\left(\frac{1-\xi}{1+\xi} \mathcal{A}_{++; 0-}^{q}-\frac{1+\xi}{1-\xi} \mathcal{A}_{-+; 0-}^{q}\right)\right.
$$$$
\left.+e^{-3 i \phi} \mathcal{A}_{-+;+-}^{q}-e^{i \phi}\left(1+\frac{4 \xi^{2}}{D^{2}\left(1-\xi^{2}\right)}\right) \mathcal{A}_{++;--}^{q}\right] \text {. }
$$

For the gluon GPDs we have

$$
\begin{aligned}
H_{1}^{g T}= & {\left[\frac{\sqrt{2} e^{-2 i \phi} \xi}{D^{2}\left(1-\xi^{2}\right)}\left(\mathcal{A}_{++;+-}^{g}-\mathcal{A}_{-+;--}^{g}\right)+\frac{e^{-3 i \phi}}{D}\left(\frac{1}{1-\xi} \mathcal{A}_{0+;+-}^{g}+\frac{1}{1+\xi} \mathcal{A}_{-+; 0-}^{g}\right)+\frac{e^{-i \phi}}{D}\left(\frac{1}{1+\xi} \mathcal{A}_{++; 0-}^{g}+\frac{1}{1-\xi} \mathcal{A}_{0+;--}^{g}\right)\right.} \\
& \left.+\frac{\sqrt{2}}{\left(1-\xi^{2}\right)}\left(e^{-4 i \phi} \mathcal{A}_{-+;+-}^{g}-\mathcal{A}_{++;--}^{g}\right)\right]
\end{aligned}
$$




$$
\begin{aligned}
& H_{2}^{g T}=\left[-\frac{\sqrt{2} e^{-2 i \phi}}{D^{2}}\left(\frac{1}{(1+\xi)^{2}}(\xi-D) \mathcal{A}_{++;+-}^{g}+\frac{1}{(1-\xi)^{2}}(\xi+D) \mathcal{A}_{-+;--}^{g}\right)+\frac{2 \sqrt{2} e^{-2 i \phi} \xi}{D^{2}\left(1-\xi^{2}\right)} \mathcal{A}_{0+; 0-}^{g}\right. \\
& -e^{-3 i \phi}\left(\frac{1}{1+\xi} \mathcal{A}_{0+;+-}^{g}-\frac{1}{1-\xi} \mathcal{A}_{-+; 0-}^{g}\right)+\frac{e^{-i \phi}}{D^{3}\left(1-\xi^{2}\right)}\left(\frac{4 \xi^{2}+D(1-\xi)^{2}}{1+\xi} \mathcal{A}_{++; 0-}^{g}\right. \\
& \left.\left.-\frac{4 \xi^{2}+D(1+\xi)^{2}}{1-\xi} \mathcal{A}_{0+;--}^{g}\right)-\frac{2 \sqrt{2} \xi\left(D\left(1+\xi^{2}\right)+2 \xi^{2}\right)}{D^{4}\left(1-\xi^{2}\right)^{2}} \mathcal{A}_{++;--}^{g}\right] \\
& H_{3}^{g T}=\left[-\frac{e^{-2 i \phi}}{4 D^{2}}\left(\frac{1-\xi}{1+\xi} \mathcal{A}_{++;+-}^{g}-\frac{1+\xi}{1-\xi} \mathcal{A}_{-+;--}^{g}\right)-\frac{e^{-i \phi}}{2 \sqrt{2} D^{3}}\left(\frac{1-\xi}{1+\xi} \mathcal{A}_{++; 0-}^{g}-\frac{1+\xi}{1-\xi} \mathcal{A}_{0+;--}^{g}\right)+\frac{\xi}{D^{4}\left(1-\xi^{2}\right)} \mathcal{A}_{++;--}^{g}\right] \\
& H_{4}^{g T}=\left[\frac{e^{-2 i \phi}}{4 D^{2}}\left(\frac{1}{1+\xi} \mathcal{A}_{++;+-}^{g}+\frac{1}{1-\xi} \mathcal{A}_{-+;--}^{g}\right)+\frac{e^{-i \phi}}{2 \sqrt{2} D^{3}}\left(\frac{1-\xi}{1+\xi} \mathcal{A}_{++; 0-}^{g}+\frac{1+\xi}{1-\xi} \mathcal{A}_{0+;--}^{g}\right)+\frac{e^{-3 i \phi}}{2 D} \mathcal{A}_{-+;+-}^{g}\right. \\
& \left.-\frac{e^{i \phi}}{2 D^{3}}\left(D^{2}-\frac{4 \xi}{1-\xi^{2}}\right) \mathcal{A}_{++;--}^{g}\right] \\
& H_{5}^{g T}=\left[\frac{e^{-2 i \phi}}{D\left(1-\xi^{2}\right)}\left(\frac{D(1-\xi)(1+2 \xi)+2 \xi^{3}}{\left(1-\xi^{2}\right)(1+\xi)} \mathcal{A}_{++;+-}^{g}+\frac{D(1+\xi)(1-2 \xi)-2 \xi^{3}}{\left(1-\xi^{2}\right)(1-\xi)} \mathcal{A}_{-+;--}^{g}+2 \xi^{2} \mathcal{A}_{0+; 0-}^{g}\right)\right. \\
& +\frac{\sqrt{2} e^{-3 i \phi} \xi^{2}}{D\left(1-\xi^{2}\right)}\left(\mathcal{A}_{0+;+-}^{g}+\mathcal{A}_{-+; 0-}^{g}\right)-\frac{\sqrt{2} e^{-i \phi}}{D^{3}\left(1-\xi^{2}\right)}\left(\frac{D(1-\xi)-2 \xi}{1+\xi} \mathcal{A}_{++; 0-}^{g}+\frac{D(1+\xi)+2 \xi}{1-\xi} \mathcal{A}_{0+;--}^{g}\right) \\
& \left.+\frac{e^{-4 i \phi}}{1-\xi^{2}} \mathcal{A}_{-+;+-}^{g}+\frac{\left(D^{2}\left(1-\xi^{2}\right)-\xi^{2}\right)\left(D^{2}+4 \xi^{2}\right)}{D^{4}\left(1-\xi^{2}\right)^{2}} \mathcal{A}_{++;--}^{g}\right] \\
& H_{6}^{g T}=\left[-\frac{e^{-2 i \phi}}{D^{2}\left(1-\xi^{2}\right)}\left(\frac{D(1-\xi)-2 \xi}{\left(1-\xi^{2}\right)(1+\xi)} \mathcal{A}_{++;+-}^{g}+\frac{D(1+\xi)+2 \xi}{\left(1-\xi^{2}\right)(1-\xi)} \mathcal{A}_{-+;--}^{g}+2 \mathcal{A}_{0+; 0-}^{g}\right)\right. \\
& +\frac{\sqrt{2} e^{-3 i \phi}}{D\left(1-\xi^{2}\right)}\left(\mathcal{A}_{0+;+-}^{g}+\mathcal{A}_{-+; 0-}^{g}\right)-\frac{\sqrt{2} e^{-i \phi} \xi^{2}}{D^{3}\left(1-\xi^{2}\right)}\left(\frac{D(1-\xi)-2 \xi}{1+\xi} \mathcal{A}_{++; 0-}^{g}+\frac{D(1+\xi)+2 \xi}{1-\xi} \mathcal{A}_{0+;--}^{g}\right) \\
& \left.+\frac{e^{-4 i \phi}}{1-\xi^{2}} \mathcal{A}_{-+;+-}^{g}+\frac{\left(D^{2}(1-\xi)-2 \xi\right)\left(D^{2}(1+\xi)+2 \xi\right)}{D^{4}\left(1-\xi^{2}\right)^{2}} \mathcal{A}_{++;--}^{g}\right] \\
& H_{7}^{g T}=\frac{1-\xi^{2}}{D^{4}} \mathcal{A}_{++;--}^{g} \\
& H_{8}^{g T}=\frac{1}{D^{2}}\left[\frac{e^{-2 i \phi}}{2}\left(-\frac{1-\xi}{1+\xi} \mathcal{A}_{++;+-}^{g}+\frac{1+\xi}{1-\xi} \mathcal{A}_{-+;--}^{g}\right)+\frac{e^{-i \phi}}{\sqrt{2} D}\left(\frac{1-\xi}{1+\xi} \mathcal{A}_{++; 0-}^{g}+\frac{1+\xi}{1-\xi} \mathcal{A}_{0+;--}^{g}\right)+\frac{2 \xi^{3}}{D^{2}\left(1-\xi^{2}\right)} \mathcal{A}_{++;--}^{g}\right] \\
& H_{9}^{g T}=\frac{1}{D^{2}}\left[\frac{e^{-2 i \phi}}{2}\left(\frac{1-\xi}{1+\xi} \mathcal{A}_{++;+-}^{g}+\frac{1+\xi}{1-\xi} \mathcal{A}_{-+;--}^{g}\right)\right. \\
& \left.+\frac{e^{-i \phi}}{\sqrt{2} D}\left(-\frac{1-\xi}{1+\xi} \mathcal{A}_{++; 0-}^{g}+\frac{1+\xi}{1-\xi} \mathcal{A}_{0+;--}^{g}\right)-\left(1+\frac{2 \xi^{2}}{D^{2}\left(1-\xi^{2}\right)}\right) \mathcal{A}_{++;--}^{g}\right] \text {. }
\end{aligned}
$$

\section{APPENDIX D: MINIMAL CONVOLUTION MODEL FOR THE DEUTERON}

In this Appendix, we outline a minimal convolution model for the deuteron GPDs. The model allows us to calculate the transversity GPDs analytically and to check certain trends seen in the full convolution model.

The minimal model starts from the following assumptions:

(i) We only include the nucleon chiral odd GPD $\bar{E}_{T}$ and put all others equal to zero. Figures 9 and 10 show that this is a reasonable starting point.

(ii) We do not include a $D$-wave component in the deuteron wave function.

(iii) We do not consider a spatial wave function for the $S$-wave. This means we only include the nucleon spin sums (through Clebsch-Gordan coefficients) and consider the following symmetric kinematics in the convolution: 


$$
\begin{array}{rlrl}
\boldsymbol{P}_{\perp} & =0, \quad \Delta^{y}=0, \quad \phi=0, & \\
\alpha_{1} & =1+\xi, & \alpha_{1}^{\prime}=1-\xi, & \\
k_{\perp}^{x} & =-\frac{\Delta^{x}}{4}, & k_{\perp}^{y}=0, \quad k_{\perp}^{\prime x}=\frac{\Delta^{x}}{4}, & k_{\perp}^{\prime y}=0, \\
\xi_{N} & =\frac{2 \xi}{1+\xi^{2}}, & & x_{N}=\frac{2 x}{1+\xi^{2}} .
\end{array}
$$

With the choice of this kinematics the symmetry constraints of Sec. II B are still obeyed.

In this minimal convolution model, we obtain for the nucleon helicity amplitudes

$$
\begin{aligned}
\int \mathrm{d} x_{N} \mathcal{A}_{++;+-}^{N}\left(x_{N}, \xi_{N}, t\right) & =\left(1-\xi_{N}\right) \frac{\sqrt{t_{0 N}-t}}{4 m} F(t), \\
\int \mathrm{d} x_{N} \mathcal{A}_{-+;--}^{N}\left(x_{N}, \xi_{N}, t\right) & =\left(1+\xi_{N}\right) \frac{\sqrt{t_{0 N}-t}}{4 m} F(t), \\
\int \mathrm{d} x_{N} \mathcal{A}_{++;--}^{N}\left(x_{N}, \xi_{N}, t\right) & =-\frac{\xi_{N}^{2}}{\sqrt{1-\xi_{N}^{2}}} F(t), \\
\int \mathrm{d} x_{N} \mathcal{A}_{-+;+-}^{N}\left(x_{N}, \xi_{N}, t\right) & =0,
\end{aligned}
$$

where $F(t)=\int \mathrm{d} x_{N} \bar{E}_{T}\left(x_{N}, \xi_{N}, t\right)$.

Using Eq. (39) in the minimal version, we obtain for the deuteron helicity amplitudes

$$
\begin{aligned}
\int \mathrm{d} x \mathcal{A}_{++;+-}^{q}(x, \xi, t) & =(1-\xi)^{2} \frac{\sqrt{t_{0 N}-t}}{2 m} F(t), \\
\int \mathrm{d} x \mathcal{A}_{-+;--}^{q}(x, \xi, t) & =(1+\xi)^{2} \frac{\sqrt{t_{0 N}-t}}{2 m} F(t), \\
\int \mathrm{d} x \mathcal{A}_{0+; 0-}^{q}(x, \xi, t) & =\left(1+\xi^{2}\right) \frac{\sqrt{t_{0 N}-t}}{2 m} F(t), \\
\int \mathrm{d} x \mathcal{A}_{0+;+-}^{q}(x, \xi, t) & =\int \mathrm{d} x \mathcal{A}_{-+; 0-}^{q}(x, \xi, t)=0, \\
\int \mathrm{d} x \mathcal{A}_{++; 0-}^{q}(x, \xi, t) & =\int \mathrm{d} x \mathcal{A}_{0+;--}^{q}(x, \xi, t) \\
& =-4 \sqrt{2} \frac{\xi^{2}}{1-\xi^{2}} F(t), \\
\int \mathrm{d} x \mathcal{A}_{++;--}^{q}(x, \xi, t) & =\int \mathrm{d} x \mathcal{A}_{-+;+-}^{q}(x, \xi, t)=0 .
\end{aligned}
$$

Note that the first moments of $\mathcal{A}^{0++-}(x, \xi, t)$ and $\mathcal{A}^{-+0-}(x, \xi, t)$ are zero because the first moment of $\mathcal{A}_{N}^{-++-}\left(x_{N}, \xi_{N}, t\right)$ is zero (which is the only one contributing to those on the nucleon level), and the first moments of $\mathcal{A}^{++--}(x, \xi, t)$ and $\mathcal{A}^{-++-}(x, \xi, t)$ are zero because we did not include a $D$-wave in the deuteron wave function.

Finally, using Eqs. (C21) to (C29), we obtain for the chiral odd quark GPDs

$$
\begin{aligned}
\int \mathrm{d} x H_{1}^{T}(x, \xi, t)= & -16 \sqrt{2} \frac{\xi^{2}}{\left(1-\xi^{2}\right)^{2}}\left(\sqrt{\frac{\left(1-\xi^{2}\right)\left(t_{0 N}-t\right)}{\left(t_{0}-t\right)}} \frac{M}{2 m}+1\right) F(t), \\
\int \mathrm{d} x H_{2}^{T}(x, \xi, t)= & -4 \sqrt{2} \frac{\xi}{\left(1-\xi^{2}\right)^{2}}\left(1+\frac{\xi^{2}}{D^{2}}\right)\left(D\left(3+\xi^{2}\right) \frac{\sqrt{t_{0 N}-t}}{2 m}+\frac{8 \xi^{2}}{1-\xi^{2}}\right) F(t), \\
\int \mathrm{d} x H_{3}^{T}(x, \xi, t)= & 4 \xi \frac{\left(1+\xi^{2}\right)}{\left(1-\xi^{2}\right)} \sqrt{\frac{\left(t_{0 N}-t\right)}{\left(t_{0}-t\right)\left(1-\xi^{2}\right)}} \frac{M}{m} F(t)-16 \frac{\xi^{3}}{D^{2}\left(1-\xi^{2}\right)^{2}} F(t), \\
\int \mathrm{d} x H_{4}^{T}(x, \xi, t)= & 2 \frac{\left(1+3 \xi^{2}\right)}{\left(1-\xi^{2}\right)} \sqrt{\frac{\left(t_{0 N}-t\right)}{\left(t_{0}-t\right)\left(1-\xi^{2}\right)}} \frac{M}{m} F(t)-8 \xi^{2} \frac{1+\xi^{2}}{D^{2}\left(1-\xi^{2}\right)^{2}} F(t), \\
\int \mathrm{d} x H_{5}^{T}(x, \xi, t)= & 16 \sqrt{2} \frac{\xi^{4}}{D^{2}\left(1-\xi^{2}\right)^{3}} F(t)+8 \sqrt{2} \xi^{2} \frac{\left(1+\xi^{2}\right)}{\left(1-\xi^{2}\right)^{3}} F(t)-\frac{\sqrt{\left(1-\xi^{2}\right)\left(t_{0}-t\right)\left(t_{0 N}-t\right)}}{\sqrt{2} M m} \frac{\left(1+3 \xi^{2}\right)}{\left(1-\xi^{2}\right)} F(t) \\
& -4 \sqrt{\frac{2\left(t_{0 N}-t\right)}{\left(t_{0}-t\right)\left(1-\xi^{2}\right)} \frac{M}{2 m} \xi^{2} \frac{(3)}{\left(1-\xi^{2}\right)^{2}} F(t),} \\
\int \mathrm{d} x H_{6}^{T}(x, \xi, t)= & -\left(1+\xi^{2}\right) \sqrt{\frac{\left(t_{0 N}-t\right)}{\left(t_{0}-t\right)\left(1-\xi^{2}\right)} \frac{M}{m}} F(t), \quad \int \mathrm{d} x H_{7}^{T}(x, \xi, t)=0, \\
\int \mathrm{d} x H_{8}^{T}(x, \xi, t)= & 16 \xi \frac{1+\xi^{2}}{1-\xi^{2}} \frac{M}{2 m} \sqrt{\frac{t_{0 N}-t}{\left(t_{0}-t\right)\left(1-\xi^{2}\right)}} F(t)+16 \frac{\xi^{3}\left(1+\xi^{2}\right)}{D^{2}\left(1-\xi^{2}\right)^{2}} F(t), \\
\int \mathrm{d} x H_{9}^{T}(x, \xi, t)= & 8 \xi^{2} \frac{3+\xi^{2}}{1-\xi^{2}} \frac{M}{2 m} \sqrt{\frac{t_{0 N}-t}{\left(t_{0}-t\right)\left(1-\xi^{2}\right)}} F(t)-\frac{32 \xi^{4}}{D^{2}\left(1-\xi^{2}\right)^{2}} F(t) .
\end{aligned}
$$


[1] M. Diehl, Phys. Rep. 388, 41 (2003).

[2] A. V. Belitsky and A. V. Radyushkin, Phys. Rep. 418, 1 (2005).

[3] S. Fucini, S. Scopetta, and M. Viviani, Phys. Rev. C 98, 015203 (2018).

[4] R. Dupré and S. Scopetta, Eur. Phys. J. A 52, 159 (2016).

[5] M. Rinaldi and S. Scopetta, Phys. Rev. C 85, 062201 (2012).

[6] S. K. Taneja, K. Kathuria, S. Liuti, and G. R. Goldstein, Phys. Rev. D 86, 036008 (2012).

[7] S. Scopetta, Phys. Rev. C 79, 025207 (2009).

[8] S. Liuti and S. K. Taneja, Phys. Rev. C 72, 032201 (2005).

[9] S. Liuti and S. K. Taneja, Phys. Rev. C 72, 034902 (2005).

[10] A. Freund and M. Strikman, Eur. Phys. J. C 33, 53 (2004).

[11] V. Guzey and M. Strikman, Phys. Rev. C 68, 015204 (2003).

[12] S. Scopetta, Phys. Rev. C 70, 015205 (2004).

[13] W. Boeglin and M. Sargsian, Int. J. Mod. Phys. E 24, 1530003 (2015).

[14] E. R. Berger, F. Cano, M. Diehl, and B. Pire, Phys. Rev. Lett. 87, 142302 (2001).

[15] A. Kirchner and D. Mueller, Eur. Phys. J. C 32, 347 (2003).

[16] F. Cano and B. Pire, Eur. Phys. J. A 19, 423 (2004).

[17] M. Mazouz et al. (Jefferson Lab Hall A Collaboration), Phys. Rev. Lett. 118, 222002 (2017).

[18] B. Pire, K. Semenov-Tian-Shansky, L. Szymanowski, and S. Wallon, Eur. Phys. J. A 50, 90 (2014).

[19] M. Diehl, Eur. Phys. J. C 19, 485 (2001).

[20] A. Yu. Umnikov, H.-x. He, and F. C. Khanna, Phys. Lett. B 398, 6 (1997).

[21] A. Bacchetta and P. J. Mulders, Phys. Rev. D 62, 114004 (2000).

[22] R. L. Jaffe and A. Manohar, Phys. Lett. B 223, 218 (1989).

[23] D. Boer, S. Cotogno, T. van Daal, P. J. Mulders, A. Signori, and Y.-J. Zhou, J. High Energy Phys. 10 (2016) 013.

[24] G. L. Li, K. F. Liu, and G. E. Brown, Phys. Lett. B 213, 531 (1988).

[25] D. F. Geesaman, K. Saito, and A. W. Thomas, Annu. Rev. Nucl. Part. Sci. 45, 337 (1995).

[26] S. A. Kulagin and R. Petti, Nucl. Phys. A765, 126 (2006).

[27] C. Ciofi degli Atti, L. L. Frankfurt, L. P. Kaptari, and M. I. Strikman, Phys. Rev. C 76, 055206 (2007).

[28] W. Cosyn, Y.-B. Dong, S. Kumano, and M. Sargsian, Phys. Rev. D 95, 074036 (2017).

[29] S. J. Brodsky, K. Y. -J. Chiu, J.-P. Lansberg, and N. Yamanakab, Phys. Lett. B 783, 287 (2018).

[30] L. L. Frankfurt and M. I. Strikman, Phys. Rep. 76, 215 (1981).

[31] B. Keister and W. Polyzou, Adv. Nucl. Phys. 20, 225 (1991).

[32] L. A. Kondratyuk and M. I. Strikman, Nucl. Phys. A426, 575 (1984).
[33] P. L. Chung, W. N. Polyzou, F. Coester, and B. D. Keister, Phys. Rev. C 37, 2000 (1988).

[34] H. J. Melosh, Phys. Rev. D 9, 1095 (1974).

[35] G. A. Miller and B.C. Tiburzi, Phys. Rev. C 81, 035201 (2010).

[36] S. J. Brodsky, H.-C. Pauli, and S. S. Pinsky, Phys. Rep. 301, 299 (1998).

[37] S. V. Goloskokov and P. Kroll, Eur. Phys. J. A 47, 112 (2011).

[38] B. Pire, L. Szymanowski, and J. Wagner, Phys. Rev. D 95, 094001 (2017).

[39] M. El Beiyad, B. Pire, M. Segond, L. Szymanowski, and S. Wallon, Phys. Lett. B 688, 154 (2010).

[40] A. D. Martin, W. J. Stirling, R. S. Thorne, and G. Watt, Eur. Phys. J. C 63, 189 (2009).

[41] R. B. Wiringa, V. G. J. Stoks, and R. Schiavilla, Phys. Rev. C 51, 38 (1995).

[42] R. Machleidt, Phys. Rev. C 63, 024001 (2001).

[43] F. Gross and A. Stadler, Phys. Rev. C 78, 014005 (2008).

[44] A. J. Freese (private communication).

[45] G. A. Miller, Phys. Rev. C 89, 045203 (2014).

[46] V. Barone, A. Drago, and P. G. Ratcliffe, Phys. Rep. 359, 1 (2002).

[47] M. Diehl, T. Gousset, and B. Pire, Phys. Rev. D 59, 034023 (1999).

[48] J. C. Collins and M. Diehl, Phys. Rev. D 61, 114015 (2000).

[49] S. Ahmad, G. R. Goldstein, and S. Liuti, Phys. Rev. D 79, 054014 (2009).

[50] G. R. Goldstein, J. O. Gonzalez Hernandez, and S. Liuti, J. Phys. G 39, 115001 (2012).

[51] B. Pire and L. Szymanowski, Phys. Rev. Lett. 115, 092001 (2015).

[52] D. Yu. Ivanov, B. Pire, L. Szymanowski, and O. V. Teryaev, Phys. Lett. B 550, 65 (2002).

[53] R. Enberg, B. Pire, and L. Szymanowski, Eur. Phys. J. C 47, 87 (2006).

[54] R. Boussarie, B. Pire, L. Szymanowski, and S. Wallon, J. High Energy Phys. 02 (2017) 054.

[55] A. V. Belitsky and D. Mueller, Phys. Lett. B 486, 369 (2000).

[56] E. R. Berger, M. Diehl, and B. Pire, Eur. Phys. J. C 23, 675 (2002).

[57] W. Armstrong et al., arXiv:1708.00888.

[58] D. Boer et al., arXiv:1108.1713.

[59] J. L. Abelleira Fernandez et al. (LHeC Study Group), J. Phys. G 39, 075001 (2012).

[60] D. E. Soper, Phys. Rev. D 5, 1956 (1972).

[61] C. E. Carlson and C.-R. Ji, Phys. Rev. D 67, 116002 (2003).

[62] S. J. Brodsky, S. Gardner, and D. S. Hwang, Phys. Rev. D 73, 036007 (2006).

[63] C. Lorcé and B. Pasquini, J. High Energy Phys. 09 (2013) 138. 